\title{
The Plane Strain Young's Modulus in Cubic Materials
}

\author{
Kevin M. Knowles ${ }^{1}$
}

Received: 19 August 2016 / Published online: 31 January 2017

(C) The Author(s) 2017. This article is published with open access at Springerlink.com

\begin{abstract}
The orientation dependence of the plane strain Young's modulus, $\tilde{E}$, of cubic materials has been analysed as a function of the direction along which a uniaxial stress is applied to a single crystal and the perpendicular direction in the single crystal along which the strain is constrained to be zero. The locus of $\tilde{E}$ in the plane perpendicular to the axis of uniaxial stress is shown to be a circle when this stress is applied along $\langle 111\rangle$. For materials with anisotropy ratios $A>1$, global minima in $\tilde{E}$ occur when the stress is applied along $\langle 001\rangle$ and when the strain along one of the two perpendicular $\langle 100\rangle$ directions is set to zero. Identical global maxima in $\tilde{E}$ are found when the stress is applied along two different families of $\langle u u w\rangle$ directions and the direction of zero strain is along either a perpendicular $\langle 1 \overline{1} 0\rangle$ or $\langle w w \overline{2 u}\rangle$ direction. For materials with $A<1$, the global maxima in $\tilde{E}$ occur when the stress is applied along $\langle 001\rangle$ and when the strain along one of the two perpendicular $\langle 100\rangle$ directions is set to zero, and identical global minima are found when the stress is applied along two different families of $\langle u u w\rangle$ directions and the direction of zero strain is along either a perpendicular $\langle 1 \overline{1} 0\rangle$ or $\langle w w \overline{2 u}\rangle$ direction.
\end{abstract}

Keywords Anisotropy · Cubic materials · Elasticity · Plane strain · Tensor algebra

Mathematics Subject Classification 74E10 $\cdot 74 \mathrm{E} 15 \cdot 74 \mathrm{~B} 05$

\section{Introduction}

There are a number of physical situations in which it is convenient to invoke the concept of plane strain in elasticity problems. In isotropic elasticity, plane strain problems are defined in terms of plane strain displacements $u_{1}$ and $u_{2}$ within an $x_{1}-x_{2}$ plane being functions only of the Cartesian coordinates $x_{1}$ and $x_{2}$, with the deformation $u_{3}$ parallel to the direction $x_{3}$ perpendicular to both $x_{1}$ and $x_{2}$ being set to zero [1]. Examples of such physical situations

K.M. Knowles

kmk10@cam.ac.uk

1 Department of Materials Science and Metallurgy, University of Cambridge, 27 Charles Babbage Road, Cambridge, CB3 OFS, UK 
given by Timoshenko and Goodier include tunnels and retaining walls with lateral pressure, as in the walls of dams [1]. Other examples where plane strain conditions are invoked are in elastic contact problems where each solid can be considered to be an elastic half-space [2], the analysis of the bending of relatively wide beams, in which the transverse bending is restricted within the central portion of the beam [3,4] and the plane strain elastic compression of polycrystalline metals prior to plastic deformation, such as in cold rolling or forging operations. If, in isotropic elasticity, $x_{1}, x_{2}$ and $x_{3}$ define the axes of the principal stresses $\sigma_{11}, \sigma_{22}$ and $\sigma_{33}$ in such situations, and if furthermore $\sigma_{22}=0$, then the ratio $\sigma_{11} / \varepsilon_{11}$ of the applied stress to the induced elastic strain parallel to the applied stress is simply $E /\left(1-v^{2}\right)$, where $E$ is Young's modulus and $v$ is Poisson's ratio [5]. This ratio is often described as the plane strain Young's modulus [6].

For an anisotropic material, Ting [7] shows that the situation is more complex than for isotropic materials, so that $u_{3}$ and the shear strains $\varepsilon_{13}$ and $\varepsilon_{23}$ do not have to be zero. However, it is still the case that the tensile strain $\varepsilon_{33}$ is zero for a condition of a generalised plane strain deformation in an anisotropic material. Therefore, as Hopcroft et al. recognise in their discussion of the plane strain Young's modulus for single crystal silicon in connection with the small deflections of short, relatively wide beams [4], the plane strain Young's modulus for a situation where $\sigma_{22}=0$ is a function of the orientation of the direction $x_{1}$ along which the stress is applied in the $x_{1}-x_{2}$ plane and the perpendicular direction $x_{3}$ along which $\varepsilon_{33}$ is zero. Hence, the plane strain Young's modulus for silicon is orientation dependent, and therefore potentially an important consideration in the design of micromechanical systems using silicon.

Interestingly, while the orientation dependence of the Young's modulus of anisotropic crystalline materials is well known [8] and the orientation dependence and extrema of Poisson's ratio in particular as function of orientation has been a subject of recent interest [9, 10], not least because of the concept of auxeticity [11], the specifics of the orientation dependence of the plane strain Young's modulus have not been addressed, even in what might be expected to be the relatively straightforward case of cubic materials such as silicon. It is the purpose of this paper to consider this orientation dependence and to identify extrema in the plane strain Young's modulus for cubic crystals as a function of the three independent elastic constants $c_{11}, c_{12}$ and $c_{44}$ in contracted Voigt notation (see the Appendix), which are themselves subject to the constraints that $c_{44}>0, c_{11}>\left|c_{12}\right|$ and $c_{11}+2 c_{12}>0$, following from the condition that the strain energy of a crystal must be positive [7, 8]. As Nye notes [8], the three unique elastic compliance constants $s_{11}, s_{12}$ and $s_{44}$ for cubic crystals are subject to identical constraints.

The paper is organised as follows. A statement of the problem of determining the plane strain Young's modulus for a plane of a general anisotropic material subject to a specific direction within that plane not being strained is given in Sect. 2 before attention is focused on the general form of the formula relevant to cubic materials. The application of this formula to directions along which a stress is applied lying in a standard stereographic triangle, or directions related to these by symmetry, is considered in detail in Sect. 3. As a result of this, extrema are identified, both within planes perpendicular to the direction of applied stress, and globally for combinations of directions of applied stress and specific directions perpendicular to this direction not being strained as a function of $c_{11}, c_{12}$ and $c_{44}$. Practical consequences of the results established in Sect. 3 are considered in Sect. 4. 


\section{General Tensor Transformation Relations for Plane Strain Young's Modulus}

Under the assumption that elastic conditions pertain, the symmetric stress and strain tensors, $\sigma_{i j}$ and $\varepsilon_{k l}$ respectively, are related to one another through the equations

$$
\sigma_{i j}=C_{i j k l} \varepsilon_{k l} \quad \text { and } \quad \varepsilon_{i j}=S_{i j k l} \sigma_{k l},
$$

in which $C_{i j k l}$ is the stiffness tensor and $S_{i j k l}$ is the compliance tensor, both tensors of the fourth rank, and where $i, j, k$ and $l$ take all values between 1 and 3 [5, 7, 8, 12, 13]. For an arbitrary rotation of axes from one axis system to another, fourth rank tensors, $T_{i j k l}$, transform as

$$
T_{i j k l}^{\prime}=a_{i m} a_{j n} a_{k p} a_{l q} T_{m n p q},
$$

for $i, j, k, l, m, n, p$ and $q$ all taking values from 1 to 3 , where the $a_{i m}$ are direction cosines specifying the angle between the $i$ th axis of the 'new' axis system and the $m$ th axis of the 'old' axis system. Both the 'old' axis system and the 'new' axis system in this formalism are defined by orthonormal axis systems. For materials with relatively high symmetry, 'old' axis systems are straightforward to define with respect to the crystal axes, whereas this is not the case with monoclinic or triclinic symmetry [13].

Defining axes $1^{\prime}, 2^{\prime}$ and $3^{\prime}$ as the axes in the 'new' axis system parallel to the axes of principal stress, with the primes to denote that these three new axes are not all aligned along the crystal axes, it is convenient to choose axis $3^{\prime}$ to be parallel to the direction along which the condition of plane strain is invoked, so that $\varepsilon_{33}^{\prime}=0$, and to choose $1^{\prime}$ to be the direction along which a stress is applied, with the stress $\sigma_{22}^{\prime}$ along $2^{\prime}$ set to zero. Hence, we have the equations

$$
\begin{aligned}
& \varepsilon_{11}^{\prime}=S_{1111}^{\prime} \sigma_{11}^{\prime}+S_{1133}^{\prime} \sigma_{33}^{\prime}, \\
& \varepsilon_{33}^{\prime}=S_{3311}^{\prime} \sigma_{11}^{\prime}+S_{3333}^{\prime} \sigma_{33}^{\prime}=0 .
\end{aligned}
$$

Using the second of these equations to substitute for $\sigma_{33}^{\prime}$ in the first of these equations, it is apparent that the plane strain Young's modulus $\tilde{E}$ parallel to the direction $1^{\prime}$ is defined by the expression

$$
\tilde{E}=\frac{\sigma_{11}^{\prime}}{\varepsilon_{11}^{\prime}}=\frac{S_{3333}^{\prime}}{S_{1111}^{\prime} S_{3333}^{\prime}-S_{1133}^{\prime} S_{3311}^{\prime}} .
$$

Using the contracted two suffix Voigt notation for the $S_{i j k l}$ (see the Appendix), this equation simplifies to the form

$$
\tilde{E}=\frac{s_{33}^{\prime}}{s_{11}^{\prime} s_{33}^{\prime}-s_{13}^{\prime 2}} .
$$

Equation (5) can be written in an equivalent form:

$$
\tilde{E}=\frac{\left(1 / s_{11}^{\prime}\right)}{\left(1-\frac{s_{13}^{\prime}}{s_{11}^{\prime}} \frac{s_{31}^{\prime}}{s_{33}^{\prime}}\right)} .
$$

Now, $1 / s_{11}^{\prime}$ is Young's modulus $E$ along the direction $1^{\prime}$ [7], while the ratios $-s_{13}^{\prime} / s_{11}^{\prime}$ and $-s_{31}^{\prime} / s_{33}^{\prime}$ both define Poisson ratios. Using the nomenclature used by Norris [9] we can define $v_{13}^{\prime}$ to be the ratio $-s_{13}^{\prime} / s_{11}^{\prime}$, corresponding to the ratio of the negative strain along 
$3^{\prime}$ to the positive strain along $1^{\prime}$ when a tensile stress is applied along $1^{\prime}$; similarly we can define $v_{31}^{\prime}$ to be the ratio $-s_{31}^{\prime} / s_{33}^{\prime}$. Hence, dropping the primes for convenience, Eq. (6) can also be rewritten conveniently in the form

$$
\tilde{E}_{1}=\frac{E_{1}}{\left(1-v_{13} v_{31}\right)},
$$

where the ' 1 ' subscripts on $E$ and $\tilde{E}$ indicate the direction along which the stress is applied. It is evident that this equation is similar to, but not the same as, the form of the equation quoted by Hopcroft et al. [4] for what they termed the 'plate modulus' in their Eq. (11) when considering the elastic deflection of short, relatively wide beams of single crystals.

For a general anisotropic material with known elastic constants, numerical manipulation of Eq. (5) enables the plane strain Young's modulus to be computed for general choices of crystallographic directions $1^{\prime}$ and $3^{\prime}$. Fortunately, for cubic materials such as silicon, there are only three independent elastic constants. This helps to simplify the algebra inherent within Eq. (5) and enables the orientation dependence of $\tilde{E}$ and the identification of extrema to be readily determined analytically for this relatively straightforward situation. Defining the 'old' axis system to be the orthonormal axis system aligned with respect to the $\langle 100\rangle$ directions of the cubic crystal, the $S_{i j k l}$ transform from axes 1,2 and 3 to the axes $1^{\prime}, 2^{\prime}$ and $3^{\prime}$ so that in general

$$
4 S_{i j k l}^{\prime}=4 s_{12} \delta_{i j} \delta_{k l}+s_{44}\left(\delta_{i k} \delta_{j l}+\delta_{i l} \delta_{j k}\right)+\left(4 s_{11}-4 s_{12}-2 s_{44}\right) a_{i u} a_{j u} a_{k u} a_{l u}
$$

[13]. Hence,

$$
\begin{aligned}
& S_{1111}^{\prime}=s_{11}^{\prime}=s_{11}-2 J\left(a_{11}^{2} a_{12}^{2}+a_{12}^{2} a_{13}^{2}+a_{13}^{2} a_{11}^{2}\right), \\
& S_{3333}^{\prime}=s_{33}^{\prime}=s_{11}-2 J\left(a_{31}^{2} a_{32}^{2}+a_{32}^{2} a_{33}^{2}+a_{33}^{2} a_{31}^{2}\right), \\
& S_{1133}^{\prime}=s_{13}^{\prime}=s_{31}^{\prime}=s_{12}+J\left(a_{11}^{2} a_{31}^{2}+a_{12}^{2} a_{32}^{2}+a_{13}^{2} a_{33}^{2}\right),
\end{aligned}
$$

where

$$
J=s_{11}-s_{12}-\frac{1}{2} s_{44}=\left(s_{11}-s_{12}\right)\left(1-\frac{1}{A}\right)
$$

and where $A$ is the anisotropy ratio [14-16]. When $A>1, J>0$, and when $A<1, J<0$. A cubic material is isotropic when $J=0$ and $A=1$; under these circumstances, it is evident from Eq. (6) that

$$
\tilde{E}=\frac{\left(1 / s_{11}\right)}{\left(1-\left(\frac{s_{12}}{s_{11}}\right)^{2}\right)} \equiv \frac{E}{\left(1-v^{2}\right)},
$$

i.e., the analytical formula for the plane strain Young's modulus of an isotropic material is produced.

\section{Directional Dependence of the Plane Strain Young's Modulus in Cubic Materials}

The first 1' directions to be considered will be [111], [001] and [110]. Two of these are directions at the corners of the standard 001-011-111 stereographic triangle. The third direction is related by symmetry to [011]. [001], [111] and [110] all lie on a great circle of a stereographic projection of a cubic material $90^{\circ}$ away from the $1 \overline{1} 0$ pole. 
We need then only then examine $1^{\prime}$ directions on the edges and within this standard stereographic triangle. However, it is convenient to examine the family of directions $\langle u u w\rangle$ between [001] and [110] and the family of directions $\langle 0 v w\rangle$ between [001] and [010] before finally examining $\langle u v w\rangle$ forms of $1^{\prime}$ within the standard stereographic triangle where $0<u<v<w$. Directions $\langle w u u\rangle$ between [011] and [111] on the side of the standard stereographic triangle are related by symmetry to $\langle u u w\rangle$ in terms of their elastic properties irrespective of the point group of the cubic material under consideration [8].

\section{$3.11^{\prime}$ Parallel to [111]}

Here,

$$
\left[a_{11}, a_{12}, a_{13}\right]=\left[\frac{1}{\sqrt{3}}, \frac{1}{\sqrt{3}}, \frac{1}{\sqrt{3}}\right],
$$

and because $3^{\prime}$ is perpendicular to [111], it follows that

$$
a_{31}+a_{32}+a_{33}=0
$$

for all possible directions $3^{\prime}$. Since the $a_{i j}$ are direction cosines,

$$
a_{31}^{2}+a_{32}^{2}+a_{33}^{2}=1
$$

After some straightforward mathematical manipulation of Eqs. (13) and (14), it is evident that

$$
a_{31}^{2} a_{32}^{2}+a_{32}^{2} a_{33}^{2}+a_{33}^{2} a_{31}^{2}=\frac{1}{4}
$$

for all possible directions $3^{\prime}$. Therefore, using Eq. (9), for this geometry, we have the compliance tensors

$$
s_{11}^{\prime}=s_{11}-\frac{2}{3} J, \quad s_{33}^{\prime}=s_{11}-\frac{1}{2} J, \quad s_{13}^{\prime}=s_{12}+\frac{1}{3} J,
$$

and so using Eq. (5), it is apparent that

$$
\tilde{E}_{[111]}=\frac{s_{11}-\frac{1}{2} J}{s_{11}^{2}-s_{12}^{2}-J\left(\frac{7}{6} s_{11}+\frac{2}{3} s_{12}\right)+\frac{2}{9} J^{2}},
$$

irrespective of which direction perpendicular to [111] is constrained to have the condition $\varepsilon_{33}^{\prime}=0$. Hence, the locus of the magnitude of $\tilde{E}$ as a function of orientation of $3^{\prime}$ within the (111) plane is a circle.

\section{$3.21^{\prime}$ Parallel to [001]}

Here,

$$
\left[a_{11}, a_{12}, a_{13}\right]=[0,0,1]
$$

and since $3^{\prime}$ is perpendicular to [001], it follows that $a_{33}=0$. Hence, using Eq. (9),

$$
s_{11}^{\prime}=s_{11}, \quad s_{33}^{\prime}=s_{11}-2 a_{31}^{2} a_{32}^{2} J, \quad s_{13}^{\prime}=s_{12},
$$


and

$$
\tilde{E}=\frac{s_{11}-2 a_{31}^{2} a_{32}^{2} J}{s_{11}^{2}-s_{12}^{2}-2 a_{31}^{2} a_{32}^{2} s_{11} J} .
$$

Now, $0 \leq 2 a_{31}^{2} a_{32}^{2} \leq 0.5$. Parameterising $a_{31}$ and $a_{32}$ as $\cos \theta$ and $\sin \theta$ within the (001) plane relative to the ' 1 ' and ' 2 ' crystal axes of the cubic material under consideration, minima in $\tilde{E}$ occur at $\theta=90^{\circ} n$ and maxima in $\tilde{E}$ occur at $\theta=\left(45^{\circ}+90^{\circ} n\right)$ for $n=0,1,2$ and 3 within the angular range $0^{\circ} \leq \theta \leq 360^{\circ}$ when $J>0$ and $A>1$, i.e., minima occur when $3^{\prime}$ is along $\langle 100\rangle$ directions and maxima occur when $3^{\prime}$ is along $\langle 110\rangle$ directions within (001). When $J<0$ and $A<1$, the converse is true.

When $3^{\prime}$ is along one of the two $\langle 100\rangle$ directions,

$$
\tilde{E}=\frac{s_{11}}{s_{11}^{2}-s_{12}^{2}}
$$

whereas when $3^{\prime}$ is along one of the two $\langle 110\rangle$ directions,

$$
\tilde{E}=\frac{s_{11}-\frac{1}{2} J}{s_{11}^{2}-s_{12}^{2}-\frac{1}{2} s_{11} J} .
$$

From Eqs. (21) and (22), the ratio of $\tilde{E}$ when $3^{\prime}$ is along $\langle 110\rangle$ to $\tilde{E}$ when $3^{\prime}$ is along $\langle 100\rangle$ in $(001)$ is

$$
1+\frac{(A-1) c_{12}^{2}}{\left(c_{11}+c_{12}\right)\left(A\left(c_{11}-c_{12}\right)+\left(c_{11}+c_{12}\right)\right)},
$$

making use of Eq. (10) and using the relationships between $s_{11}, s_{12}, c_{11}$ and $c_{12}$ given in the Appendix. It is of interest to calculate this ratio for the highly elastically anisotropic cubic close packed metal $\mathrm{In}-27$ at $\% \mathrm{Tl}$ as it is cooled down to its martensitic transformation temperature of $125 \mathrm{~K}$. In this phase of this material, the [110], [1̄ㅣ] polarised acoustic mode softens progressively as the martensitic transformation temperature is approached [17]. At $290 \mathrm{~K}$ where $c_{11}=39.4 \mathrm{GPa}, c_{12}=38.7 \mathrm{GPa}$ and $A=23.9$, this ratio is 5.64 ; at $200 \mathrm{~K}$ where $c_{11}=40.17 \mathrm{GPa}, c_{12}=39.97 \mathrm{GPa}$ and $A=90.7$, this ratio increases to 19.2.

\section{$3.31^{\prime}$ Parallel to [110]}

Here,

$$
\left[a_{11}, a_{12}, a_{13}\right]=\left[\frac{1}{\sqrt{2}}, \frac{1}{\sqrt{2}}, 0\right]
$$

and since $3^{\prime}$ is perpendicular to [110], it follows that $a_{32}=-a_{31}$. Hence, using Eq. (9),

$$
s_{11}^{\prime}=s_{11}-\frac{J}{2}, \quad s_{33}^{\prime}=s_{11}-2 a_{31}^{2} J\left(a_{31}^{2}+2 a_{33}^{2}\right), \quad s_{13}^{\prime}=s_{12}+a_{31}^{2} J,
$$

enabling the locus of the locus of the magnitude of $\tilde{E}$ as a function of orientation of $3^{\prime}$ within the (110) plane to be determined straightforwardly computationally.

Identification of extrema in $\tilde{E}$ as a function of $3^{\prime}$ within (110) can be made by parameterising $\left[a_{31}, a_{32}, a_{33}\right]$ so that this unit vector is written in the form

$$
\left[a_{31}, a_{32}, a_{33}\right]=\left[\frac{\cos \theta}{\sqrt{2}},-\frac{\cos \theta}{\sqrt{2}},-\sin \theta\right]
$$


and then considering extrema in

$$
\frac{1}{\tilde{E}}=s_{11}^{\prime}-\frac{s_{13}^{\prime 2}}{s_{33}^{\prime}}
$$

with

$$
s_{11}^{\prime}=s_{11}-\frac{J}{2}, \quad s_{33}^{\prime}=s_{11}-2 J\left(\cos ^{2} \theta-\frac{3}{4} \cos ^{4} \theta\right), \quad s_{13}^{\prime}=s_{12}+J \frac{\cos ^{2} \theta}{2} .
$$

Since only $s_{33}^{\prime}$ and $s_{13}^{\prime}$ are functions of $\theta$ here, it follows that

$$
\frac{\mathrm{d}}{\mathrm{d} \theta}\left(\frac{1}{\tilde{E}}\right)=\frac{s_{13}^{\prime}}{s_{33}^{\prime 2}}\left(s_{13}^{\prime} \frac{\mathrm{d}}{\mathrm{d} \theta}\left(s_{33}^{\prime}\right)-2 s_{33}^{\prime} \frac{\mathrm{d}}{\mathrm{d} \theta}\left(s_{13}^{\prime}\right)\right) .
$$

Therefore, turning points in $1 / \tilde{E}$ occur when

$$
\frac{s_{13}^{\prime} J \sin \theta \cos \theta}{s_{33}^{\prime 2}}\left(s_{33}^{\prime}-\left(3 \cos ^{2} \theta-2\right) s_{13}^{\prime}\right)=0
$$

For anisotropic cubic materials, $J \neq 0$. Therefore, turning points in $1 / \tilde{E}$ occur when $\theta=$ $90^{\circ} n$ for integer $n=0-4$ within the angular range $0^{\circ} \leq \theta \leq 360^{\circ}$, i.e., along $\langle 1 \overline{1} 0\rangle$ and $\langle 001\rangle$ directions within (110). For cubic materials with $A>1$, the value of $\tilde{E}$ when $3^{\prime}$ is along $\langle 001\rangle$ is greater than when $3^{\prime}$ is along $\langle 1 \overline{1} 0\rangle$; the reverse is true for cubic materials with $A<1$.

Further turning points also occur if either

$$
s_{33}^{\prime}-\left(3 \cos ^{2} \theta-2\right) s_{13}^{\prime}=0
$$

or

$$
s_{13}^{\prime}=0 \text {. }
$$

Substituting for $s_{33}^{\prime}$ and $s_{13}^{\prime}$ from Eq. (28), the condition given by Eq. (31) becomes

$$
\cos ^{2} \theta=\frac{s_{11}+2 s_{12}}{J+3 s_{12}}=\frac{2 s_{11}+4 s_{12}}{2 s_{11}+4 s_{12}-s_{44}}=\frac{2 c_{44}}{2 c_{44}-\left(c_{11}+2 c_{12}\right)} .
$$

Since both $c_{44}>0$ and $\left(c_{11}+2 c_{12}\right)>0$, it is immediately apparent that this equation cannot be satisfied for real values of $\theta$ when $1^{\prime}$ is parallel to [110].

The condition given by Eq. (32) is satisfied if

$$
\cos ^{2} \theta=-\frac{2 s_{12}}{J}=-\frac{4 s_{12}}{2 s_{11}-2 s_{12}-s_{44}}=\frac{4 c_{12} c_{44}}{\left(c_{11}+2 c_{12}\right)\left(2 c_{44}+c_{12}-c_{11}\right)},
$$

in which case the value of $\tilde{E}$ is simply $1 / s_{11}^{\prime}$, i.e., identical to the Young's modulus in the $1^{\prime}$ direction. This is quite a restrictive condition because it requires the strain along $3^{\prime}$ to be zero when a stress is applied along $1^{\prime}$. Values of $\theta$ at which Eq. (32) is satisfied are necessarily minima in the magnitude of $\tilde{E}$ as a function of $\theta$ on the (110) plane.

For there to be solutions for $\theta$ from Eq. (34),

$$
0<\frac{4 c_{12} c_{44}}{\left(c_{11}+2 c_{12}\right)\left(2 c_{44}+c_{12}-c_{11}\right)}<1 .
$$


Since $c_{44}>0$ and $c_{11}+2 c_{12}>0$, the left-hand inequality is equivalent to the condition

$$
\frac{c_{12}}{2 c_{44}+c_{12}-c_{11}}>0 \text {. }
$$

Hence, if $c_{12}>0$, a necessary condition from this inequality is that we require $A>1$ for there to be solutions of Eq. (34). Likewise, if $c_{12}<0$, a necessary condition from this inequality is that we require $A<1$.

For $c_{12}>0$ and $A>1$, the right-hand inequality is equivalent to the condition

$$
4 c_{12} c_{44}<\left(c_{11}+2 c_{12}\right)\left(2 c_{44}+c_{12}-c_{11}\right) \text {, }
$$

which reduces to the condition

$$
1+\frac{2 c_{12}}{c_{11}}<A
$$

after some elementary rearrangement of Eq. (37). For $c_{12}<0$ and $A<1$, the right-hand inequality is equivalent to the condition

$$
-4 c_{12} c_{44}<-\left(c_{11}+2 c_{12}\right)\left(2 c_{44}+c_{12}-c_{11}\right),
$$

i.e.,

$$
1+\frac{2 c_{12}}{c_{11}}>A .
$$

Almost all cubic materials satisfy the condition $c_{12}>0$. For these materials, there will only be a relatively small group with $A>1$ for which Eq. (38) is also satisfied. Thus, for a moderately anisotropic material such as $\mathrm{Si}$, where $c_{11}=165.7 \mathrm{GPa}, c_{12}=63.9 \mathrm{GPa}, c_{44}=$ $79.6 \mathrm{GPa}$ and $A=1.56$ (Table 1), there is no real solution for $\theta$ from Eq. (34). However, for $\mathrm{Cu}$, where $c_{11}=168.4 \mathrm{GPa}, c_{12}=121.4 \mathrm{GPa}, c_{44}=75.4 \mathrm{GPa}$ and $A=3.21$ (Table 1), real solutions for $\theta$ occur when $\cos \theta=0.9262$, i.e., for $\theta= \pm 22.15^{\circ}$ and $\pm 157.85^{\circ}$ within the angular range $-180^{\circ} \leq \theta \leq 180^{\circ}$. Similarly, real solutions occur for Eq. (34) for even more anisotropic materials with $A>1$ such as $\beta$-brass and the cubic close packed form of $\mathrm{In}-27$ at $\% \mathrm{Tl}$ just above the temperature at which it undergoes a martensitic transformation.

As Grimvall [18] and Brańka et al. [11] note, cubic materials with $c_{12}<0$ are very rare. However, there is strong experimental evidence that such materials do indeed exist in families of cubic rare earth chalcogenides in the intermediate valence state as a consequence of the special electronic structure of these materials and the possibility of electron transfer from $4 f$ to $5 d$ shells in the rare earth atoms as a consequence of applying a uniaxial pressure [1923 ]. Electrons in the $5 d$ shell screen the positively charged rare earth ion nucleus less well than electrons in the $4 f$ shell, so that this will cause the whole electronic shell of the rare earth ion to shrink, in effect moving it from a divalent state towards a trivalent state. When a uniaxial pressure is applied to a single crystal of one of these rare earth chalcogenides, this change in valence of the rare earth ions caused by the uniaxial pressure has the effect of shrinking the crystal in all directions because of the smaller ion radius of the $3+$ rare earth ion in comparison with that of the $2+$ rare earth ion [24].

Of the materials and compositions quoted in Refs. [19-23] with $c_{12}<0$, there are five compositions at room temperature and pressure for which Eq. (40) is satisfied: $\operatorname{Sm}_{0.58} \mathrm{Y}_{0.42} \mathrm{~S}$, $\mathrm{Tm}_{0.99} \mathrm{Se}, \mathrm{Sm}_{0.9} \mathrm{La}_{0.1} \mathrm{~S}$ and $\mathrm{Sm}_{0.85} \mathrm{Tm}_{0.15} \mathrm{~S}$. Other compositions in these materials are completely auxetic, as Brańka et al. have discussed [11], and so for these compositions Eq. (32) cannot be satisfied. 
Table 1 Elastic constants $c_{11}, c_{12}$ and $c_{44}$ (in $\mathrm{GPa}$ ) and anisotropy ratio, $A$, for $\mathrm{Sm}_{0.58} \mathrm{Y}_{0.42} \mathrm{~S}, \mathrm{Nb}, \mathrm{Si}, \mathrm{Cu}$, $\beta$-brass, and In -27 at\% Tl at $290 \mathrm{~K}$. Data for $c_{11}, c_{12}$ and $c_{44}$ are from Table 1 of [21] for $\mathrm{Sm}_{0.58} \mathrm{Y}_{0.42} \mathrm{~S}$, Table 6.1 of [16] for $\mathrm{Nb}, \mathrm{Si}$ and $\mathrm{Cu}$, Table III of [25] for $\beta$-brass and Table 1 of [17] for In -27 at\% $\mathrm{Tl}$ at $290 \mathrm{~K} . s_{11}, s_{12}$ and $s_{44}$ can be determined from $c_{11}, c_{12}$ and $c_{44}$ using equations stated in the Appendix

\begin{tabular}{lcccc}
\hline Material & $c_{11}$ & $c_{12}$ & $c_{44}$ & $A$ \\
\hline $\mathrm{Sm}_{0.58} \mathrm{Y}_{0.42} \mathrm{~S}$ & 143 & -33 & 34.7 & 0.39 \\
$\mathrm{Nb}$ & 245.6 & 138.7 & 29.3 & 0.55 \\
$\mathrm{Si}$ & 165.7 & 63.9 & 79.6 & 1.56 \\
$\mathrm{Cu}$ & 168.4 & 121.4 & 75.4 & 3.21 \\
$\beta$-brass & 129.1 & 109.7 & 82.4 & 8.49 \\
$\mathrm{In}-27$ at\% $\mathrm{Tl}$ at $290 \mathrm{~K}$ & 39.4 & 38.7 & 8.38 & 23.9 \\
\hline
\end{tabular}

\subsection{Graphical Representations of the Loci of $\tilde{E}$ for 1' Parallel to (i) [001], (ii) $[111]$ and (iii) $[110]$}

To illustrate how anisotropy affects the plane strain Young's modulus of cubic materials for 1' parallel to [001], [111] and [110], six different cubic materials have been selected: $\mathrm{Sm}_{0.58} \mathrm{Y}_{0.42} \mathrm{~S}, \mathrm{Nb}, \mathrm{Si}, \mathrm{Cu}, \beta$-brass, and $\mathrm{In}-27$ at $\% \mathrm{Tl}$ at $290 \mathrm{~K}$. Data for $c_{11}, c_{12}$ and $c_{44}$ and $A$ for these six materials are shown in Table 1. For each material, loci of $\tilde{E}$ as a function of orientation within the plane perpendicular to $1^{\prime}$ are shown in Fig. 1 plotted with a common horizontal [1 $1 \overline{1} 0$ ] direction. For these three different choices of the $1^{\prime}$ direction, this form of graphical representation has the advantage of identifying visually maxima and minima in $\tilde{E}$, both for a specific choice of direction and for comparison with the other two choices of direction.

It is evident from Fig. 1 that the loci for [001] and [110] have $4 \mathrm{~mm}$ and $\mathrm{mm}$ symmetry respectively at the origin, so that both have mirror planes parallel to the common horizontal [11 10 ] direction and the relevant vertical directions. Hence, attention need only be focused on the angular range $0^{\circ} \leq \theta \leq 90^{\circ}$; for convenience when specifying angles, $\theta$ can be chosen to be positive when the angle turned through is anticlockwise relative to the common horizontal [110] direction.

$\mathrm{Sm}_{0.58} \mathrm{Y}_{0.42} \mathrm{~S}$ is a material for which $c_{12}<0$ and $A<1$. Hence, in Fig. 1(a), for this material, the circular locus of $\tilde{E}$ for $1^{\prime}$ parallel to [111] (in black) lies entirely within the locus of $\tilde{E}$ for $1^{\prime}$ parallel to [001] (in red). This is also a material for which Eq. (34) is satisfied when $1^{\prime}$ is parallel to [011] at $\theta=41.67^{\circ}$. However, the effect is very subtle: when $\theta=0^{\circ}$, $\tilde{E}=82.08 \mathrm{GPa}$; when $\theta=41.67^{\circ}, \tilde{E}=80.12 \mathrm{GPa}$, and when $\theta=0^{\circ}, \tilde{E}=85.11 \mathrm{GPa}$. It is best appreciated by calibrating the locus in blue of $\tilde{E}$ for $1^{\prime}$ parallel to [011] relative to that in black for $1^{\prime}$ parallel to [111] and recognising that the blue and black loci are most close along an extended radius of the black circle when $\theta$ is close to the bisector of the horizontal and vertical axes. A further feature of the locus in red of $\tilde{E}$ for $1^{\prime}$ parallel to [001] for $\mathrm{Sm}_{0.58} \mathrm{Y}_{0.42} \mathrm{~S}$ is the relatively small deviation from unity of the ratio of $\tilde{E}$ when $3^{\prime}$ is along $\langle 110\rangle$ to $\tilde{E}$ when $3^{\prime}$ is along $\langle 100\rangle$ : this ratio is 0.967 . This small deviation can be rationalised straightforwardly because of the relatively small magnitude of $c_{12}$. In other cubic materials with $A<0$ and relatively small positive $c_{12}$, the effect is also small: thus, for $\mathrm{KCl}$ using the values of $c_{11}, c_{12}$ and $c_{44}$ in Table III of [25], the ratio is 0.989 .

The effect of a relatively large and positive $c_{12}$ when $A<1$ is demonstrated by the graphical representations in Fig. 1(b) for Nb. The locus of $\tilde{E}$ for $1^{\prime}$ parallel to [110] now only has a single maximum $\theta=0^{\circ}$ at $0^{\circ}$ and a single minimum at $90^{\circ}$ within the angular range 


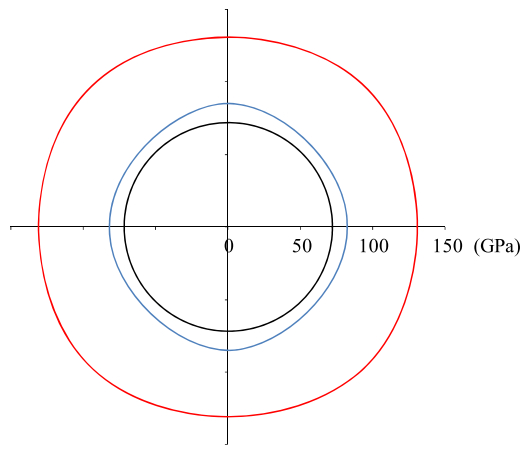

(a)

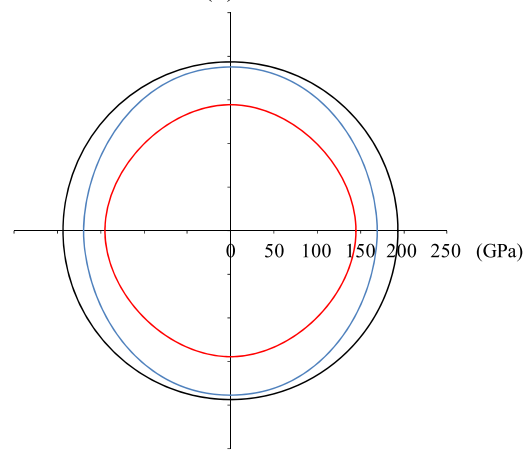

(c)

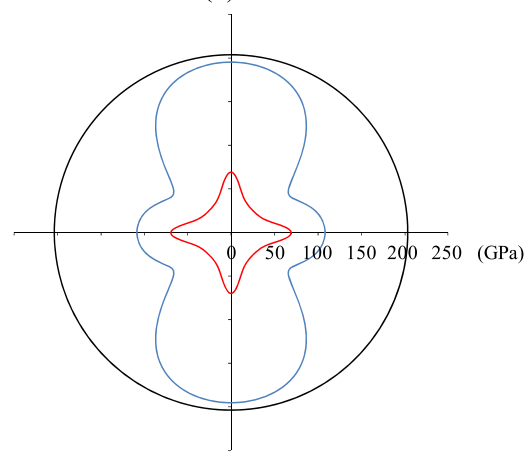

(e)

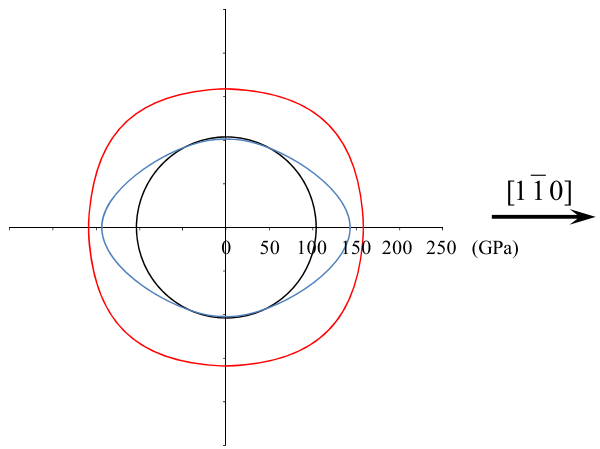

(b)

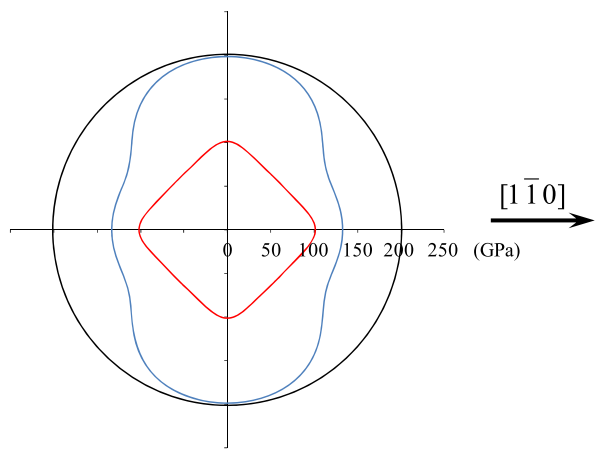

(d)

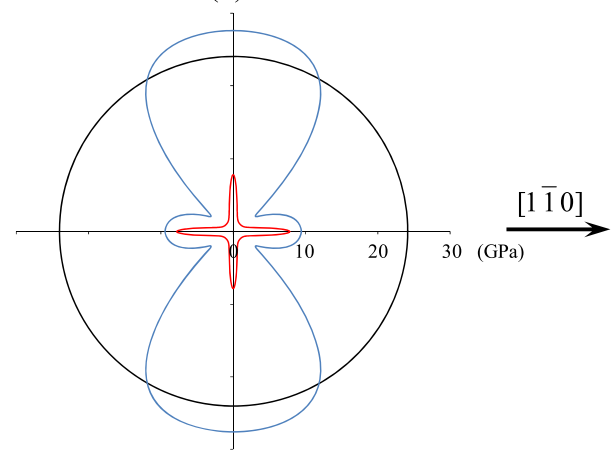

(f)

Fig. 1 Loci of $\tilde{E}$ for $1^{\prime}$ parallel to [001] (in red), [111] (in black) and [110] (in blue) for (a) $\mathrm{Sm}_{0.58} \mathrm{Y}_{0.42} \mathrm{~S}$, (b) $\mathrm{Nb}$, (c) $\mathrm{Si}$, (d) $\mathrm{Cu}$, (e) $\beta$-brass and (f) $\mathrm{In}-27$ at\% $\mathrm{Tl}$ at $290 \mathrm{~K}$. In each diagram the horizontal direction is [1 $1 \overline{10}$ ]. The vertical directions are [110] when $1^{\prime}$ is parallel to [001], [112] ] when $1^{\prime}$ is parallel to [111] and [001] when $1^{\prime}$ is parallel to [110]. (Colour figure online)

$0^{\circ} \leq \theta \leq 90^{\circ}$. For the values of $c_{11}, c_{12}$ and $c_{44}$ for $\mathrm{Nb}$, it is noteworthy that $\tilde{E}$ for $1^{\prime}$ parallel to [110] and $3^{\prime}$ parallel to [00 $\left.\overline{1}\right]$ is slightly less than when $1^{\prime}$ parallel to [111]. Indeed, a trend seen in all six graphical representations in Fig. 1 is that these two values are similar.

A comparison of Eq. (17) and Eq. (5) with the $s_{11}^{\prime}, s_{33}^{\prime}$ and $s_{13}^{\prime}$ given in Eq. (28) when $\theta=90^{\circ}$ shows after some algebra that these two forms for $\tilde{E}$ are formally identical when 


$$
s_{44}=\frac{2\left(7 s_{11}+9 s_{12}\right)\left(s_{11}+2 s_{12}\right)}{s_{11}},
$$

or, equivalently,

$$
c_{44}=\frac{\left(c_{11}+c_{12}\right)\left(c_{11}+2 c_{12}\right)}{2\left(7 c_{11}-2 c_{12}\right)} .
$$

Furthermore, since $A<1$ for $\mathrm{Nb}$, the locus for $\tilde{E}$ for $1^{\prime}$ parallel to [001] for Nb shows noticeably higher values of $\tilde{E}$ along $\langle 100\rangle$ than along $\langle 110\rangle$, as a consequence of the relatively large and positive $c_{12}$.

For a material with $A>1$ such as Si with a moderate degree of anisotropy $(A=1.56)$, the locus of $\tilde{E}$ for $1^{\prime}$ parallel to [001] is relatively circular-when $3^{\prime}$ is parallel to $\langle 110\rangle \tilde{E}=$ $144.7 \mathrm{GPa}$, while $\tilde{E}=141.1 \mathrm{GPa}$ when $3^{\prime}$ is parallel to $\langle 100\rangle$. It is only the locus for $1^{\prime}$ parallel to [110] which shows a clear deviation from a circle: when $3^{\prime}$ is parallel to $\langle 110\rangle$, $\tilde{E}=169.8 \mathrm{GPa}$, while $\tilde{E}=188.0 \mathrm{GPa}$ when $3^{\prime}$ is parallel to $\langle 001\rangle$.

When materials with noticeable anisotropy and extreme anisotropy are considered, such as $\mathrm{Cu}, \beta$-brass and $\mathrm{In}-27$ at $\% \mathrm{Tl}$ at $290 \mathrm{~K}$ in Figs. 1(d)-(f) respectively, it is apparent that the loci of $\tilde{E}$ for $1^{\prime}$ parallel to [001] have strong angular dependencies, so that in the extreme example of In -27 at $\% \mathrm{Tl}$ at $290 \mathrm{~K}$ it has a very low stiffness when $3^{\prime}$ is parallel to $\langle 100\rangle$ ( $\tilde{E}=1.4 \mathrm{GPa})$ and is noticeably more stiff when $3^{\prime}$ is parallel to $\langle 110\rangle(\tilde{E}=7.8 \mathrm{GPa})$. It is stiffer still when $1^{\prime}$ is parallel to [111] $(\tilde{E}=24.1 \mathrm{GPa})$ and most stiff for these choices of $1^{\prime}$ when $1^{\prime}$ is parallel to [110] and $3^{\prime}$ is parallel to $\langle 001\rangle(\tilde{E}=27.6 \mathrm{GPa})$. For all three of these materials, the loci of $\tilde{E}$ when $1^{\prime}$ is parallel to [110] have angles where Eq. (32) is satisfied, clearly identifiable in all three examples by the minima in the magnitude of the radius vector from the origin as a function of angle.

\section{$3.51^{\prime}$ Parallel to $\langle u u w\rangle$ Between [001] and [110]}

The loci of $\tilde{E}$ for three specific choices of [001], [111] and [110] for $1^{\prime}$ in Fig. 1 as a function of the elastic constants are special cases of a more general form for $1^{\prime}$ of the form $[u u w]$. For such a direction, we can parameterise $\left[a_{11}, a_{12}, a_{13}\right]$ as

$$
\left[a_{11}, a_{12}, a_{13}\right]=\left[\frac{\sin \varphi}{\sqrt{2}}, \frac{\sin \varphi}{\sqrt{2}}, \cos \varphi\right] .
$$

For each choice of $1^{\prime}$, it is convenient to have [1 $\left.\overline{1} 0\right]$ as a common horizontal direction, as in the graphical representations in Fig. 1, in order to visualise how the loci of $\tilde{E}$ change as a function of angle $\varphi$ from [001] towards [110].

Hence, relative to the crystallographic set of orthonormal axes $x_{1}, x_{2}$ and $x_{3}$, we can define a new orthonormal right-hand axis set $\beta_{1}, \beta_{2}$ and $\beta_{3}$, so that the table of direction cosines between the axis set 1,2 and 3 and this new axis set will be of the form

$$
\begin{array}{c|ccc}
\multicolumn{1}{c}{} & x_{1} & x_{2} & x_{3} \\
\cline { 2 - 4 } x_{\beta_{1}}^{\prime} & \frac{\sin \varphi}{\sqrt{2}} & \frac{\sin \varphi}{\sqrt{2}} & \cos \varphi \\
x_{\beta_{2}}^{\prime} & \frac{-\cos \varphi}{\sqrt{2}} & \frac{-\cos \varphi}{\sqrt{2}} & \sin \varphi \\
x_{\beta_{3}}^{\prime} & \frac{1}{\sqrt{2}} & -\frac{1}{\sqrt{2}} & 0
\end{array},
$$

with the primes in Eq. (44) denoting that these three new $\beta$ axes are not aligned along the $\langle 100\rangle$ crystal axes. Therefore, with respect to $\beta_{2}$ and $\beta_{3}$, a direction of unit length making 
anticlockwise angles of $\left(90^{\circ}+\theta\right)$ with $\beta_{2}$ and $\theta$ with $\beta_{3}$ along which there is a condition of plane strain will have direction cosines

$$
\left[a_{31}, a_{32}, a_{33}\right]=\left[\frac{\cos \theta+\sin \theta \cos \varphi}{\sqrt{2}}, \frac{-\cos \theta+\sin \theta \cos \varphi}{\sqrt{2}},-\sin \theta \sin \varphi\right] .
$$

Using Eq. (9), it is apparent for this geometry that we have the three compliance tensors

$$
\begin{aligned}
& s_{11}^{\prime}=s_{11}-\frac{J \sin ^{2} \varphi}{2}\left(4-3 \sin ^{2} \varphi\right), \\
& s_{33}^{\prime}=s_{11}-\frac{J}{2}\left(1+\sin ^{2} \theta\left(6 \sin ^{2} \varphi-4\right)+\sin ^{4} \theta\left(4-4 \sin ^{2} \varphi-3 \sin ^{4} \varphi\right)\right), \\
& s_{13}^{\prime}=s_{12}+\frac{J \sin ^{2} \varphi}{2}\left(1+\sin ^{2} \theta\left(2-3 \sin ^{2} \varphi\right)\right) .
\end{aligned}
$$

For a specific value of $\varphi$, turning points in $1 / \tilde{E}$ occur when Eq. (29) is zero, i.e., when

$$
\frac{s_{13}^{\prime} J \sin \theta \cos \theta\left(1-3 \cos ^{2} \varphi\right)}{s_{33}^{\prime 2}} f(\theta)=0,
$$

where

$$
f(\theta)=s_{33}^{\prime} \sin ^{2} \varphi-s_{13}^{\prime}\left(1-\sin ^{2} \theta\left(2+\sin ^{2} \varphi\right)\right) .
$$

Thus, for example, when $\varphi=0^{\circ}$, i.e., when [uuw] is [001], turning points occur in the angular range $0^{\circ} \leq \theta \leq 90^{\circ}$ at $\theta=0^{\circ}$ and $90^{\circ}$ from the conditions $\sin \theta=0$ and $\cos \theta=0$ respectively, and when $\theta=45^{\circ}$ from the condition $f(\theta)=0$. At this orientation, the condition $s_{13}^{\prime}=0$ is only satisfied if, by chance, $s_{12}=0$ (Eq. (19)); this in turn would require $c_{12}=0$ for the material under consideration.

When $\varphi=90^{\circ}$, i.e., when [uuw] is [110], turning points occur in the angular range $0^{\circ} \leq \theta \leq 90^{\circ}$ at $\theta=0^{\circ}$ and $90^{\circ}$ from the conditions $\sin \theta=0$ and $\cos \theta=0$ respectively. In addition, turning points occur for materials for which the condition $s_{13}^{\prime}=0$ is satisfied (Sect. 3.3), but there are no real values of $\theta$ for which Eq. (48) is satisfied-this is then identical to the condition expressed by Eq. (31).

When $\varphi=\cos ^{-1}(1 / \sqrt{3})$, i.e., when [uuw] is [111], it is evident that Eq. (47) is also satisfied. At this orientation the locus of $\tilde{E}$ is a circle (Sect. 3.1), and so this is the only solution to Eq. (47) — there are no additional turning points as solutions.

Overall, irrespective of the precise values of the elastic constants of the cubic material under consideration, loci of $\tilde{E}$ as a function of $\theta$ have turning points in the angular range $0^{\circ} \leq \theta \leq 90^{\circ}$ at $\theta=0^{\circ}$ and $90^{\circ}$ which can be either local or global minima and maxima within the plane $(u u w)$ for the choice of parameterisations expressed by Eqs. (43) and (45), unless $\varphi=\cos ^{-1}(1 / \sqrt{3})$.

There are additional turning points in $\tilde{E}$ determined by $f(\theta)=0$; again, these occur irrespective of the precise values of the elastic constants of the cubic material under consideration. Substituting for $s_{13}^{\prime}$ and $s_{33}^{\prime}$ from Eq. (46) into Eq. (48), and rearranging, these additional turning points occur at values of $\theta$ determined by the condition

$$
\sin ^{2} \theta=\frac{s_{12}+\left(J-s_{11}\right) \sin ^{2} \varphi}{2 s_{12}+\left(2 J+s_{12}\right) \sin ^{2} \varphi-J \sin ^{4} \varphi} .
$$


Thus, for example, when $\varphi=0^{\circ}$, i.e., when $1^{\prime}$ is along [001], these turning points occur at values of $\sin ^{2} \theta=1 / 2$, i.e., along $\langle 100\rangle$ directions. Since $\sin ^{2} \theta$ cannot be greater than 1 , it is evident that this equation also defines a limiting condition for $\varphi$ above which the turning points determined by $f(\theta)=0$ disappear. This limiting value of $\varphi, \varphi_{\text {crit }}$, is determined by the condition

$$
J \sin ^{4} \varphi_{\text {crit }}-\left(s_{11}+s_{12}+J\right) \sin ^{2} \varphi_{\text {crit }}-s_{12}=0 .
$$

Thus, for example, for the highly anisotropic material $\mathrm{In}-27$ at $\% \mathrm{Tl}$ at $290 \mathrm{~K}, \varphi_{\text {crit }}=35.82^{\circ}$, i.e., close to where $1^{\prime}$ is along [112]. At this value of $\varphi_{\text {crit }}$, the condition $f(\theta)=0$ has a solution of $\theta=90^{\circ}$, i.e., the solution is coincident with a known turning point from Eq. (47).

Finally, for those cubic materials where the condition $s_{13}^{\prime}=0$ is satisfied, local minima in $\tilde{E}$ also occur. Using Eq. (46), it is apparent that such local minima occur at values of $\theta$ for allowed values of $\varphi$ for which

$$
\sin ^{2} \theta=\frac{J \sin ^{2} \varphi+2 s_{12}}{J \sin ^{2} \varphi\left(3 \sin ^{2} \varphi-2\right)} .
$$

Thus, for example, for In -27 at $\% \mathrm{Tl}$ at $290 \mathrm{~K}$, valid solutions of Eq. (51) occur for $36.90^{\circ} \leq$ $\varphi \leq 53.10^{\circ}$ and also for $56.26^{\circ} \leq \varphi \leq 90^{\circ}$. At $\varphi=36.90^{\circ}$ and at $\varphi=53.10^{\circ}, \theta=90^{\circ}$ and at $\varphi=56.26^{\circ}, \theta=0^{\circ}$, so that for this material there is a small angular range around $(u u w)=(111)$ where there is no solution to the equation $s_{13}^{\prime}=0$, and a significantly larger angular range $0^{\circ} \leq \varphi \leq 36.90^{\circ}$ where there is also no solution to the equation $s_{13}^{\prime}=0$.

The consequences of these calculations for the forms of the loci for $\tilde{E}$ on selected (uuw) planes for $0^{\circ} \leq \varphi \leq 90^{\circ}$ are shown in Figs. 2 and 3 respectively for the six materials for which stiffness coefficients are given in Table 1. The angular range (001)-(111) is covered in Fig. 2 and the angular range (111)-(110) is covered in Fig. 3. Since for some of the materials, the loci for neighbouring ( $u u w$ ) planes are very similar, some of the loci have been removed for clarity in both Fig. 2 and Fig. 3. All these (uuw) loci have the $\mathrm{mm}$ symmetry noted at the origin for [001] and [110] in Sect. 3.4, with mirror planes parallel and perpendicular to [1ํㅣㅇ.

It is evident from these two figures that for materials with $A>1$, and in particular Fig. 2(f) and Fig. 3(f) for In -27 at $\% \mathrm{Tl}$ at $290 \mathrm{~K}$, values of $\tilde{E}$ occur for these (uuw) planes along directions both parallel and perpendicular to [1 10$]$ which exceed the value of $\tilde{E}$ obtained when $1^{\prime}$ is parallel to [111]. It is therefore relevant to determine the values of $\varphi$ for which turning points in $\tilde{E}$ occur when $\theta=0^{\circ}$ and $90^{\circ}$ given the forms of $s_{11}^{\prime}, s_{33}^{\prime}$ and $s_{13}^{\prime}$ in Eq. (46).

For $\theta=0^{\circ}$, these three compliance terms are

$$
s_{11}^{\prime}=s_{11}-\frac{J}{2}\left(4 \sin ^{2} \varphi-3 \sin ^{4} \varphi\right), \quad s_{33}^{\prime}=s_{11}-\frac{J}{2} \quad \text { and } \quad s_{13}^{\prime}=s_{12}+\frac{J \sin ^{2} \varphi}{2},
$$

and so within the angular range $0^{\circ} \leq \varphi \leq 90^{\circ}$, turning points occur in $\tilde{E}$ when $\varphi=0^{\circ}$ and $90^{\circ}$, and when $\varphi$ satisfies the condition

$$
\sin ^{2} \varphi=\frac{2 s_{11}+s_{12}-J}{3 s_{11}-2 J} \equiv \frac{2 s_{11}+4 s_{12}+s_{44}}{2\left(s_{11}+2 s_{12}+s_{44}\right)} .
$$

At the critical value of $\varphi$ defined by Eq. (53) $\tilde{E}$ simplifies after some algebra to the expression

$$
\tilde{E}=\frac{4\left(s_{11}+2 s_{12}+s_{44}\right)}{s_{44}\left(4 s_{11}+8 s_{12}+s_{44}\right)}=\frac{4 c_{44}\left(c_{11}+2 c_{12}+c_{44}\right)}{c_{11}+2 c_{12}+4 c_{44}},
$$




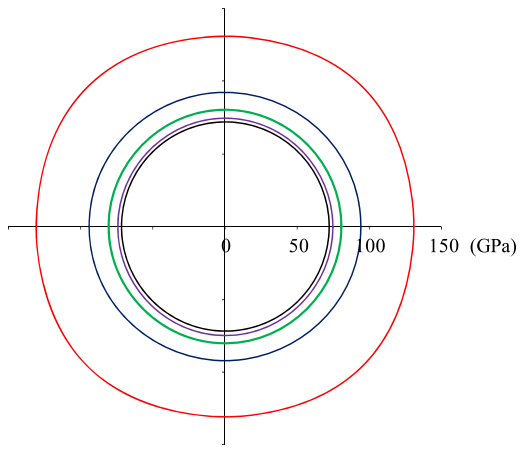

(a)

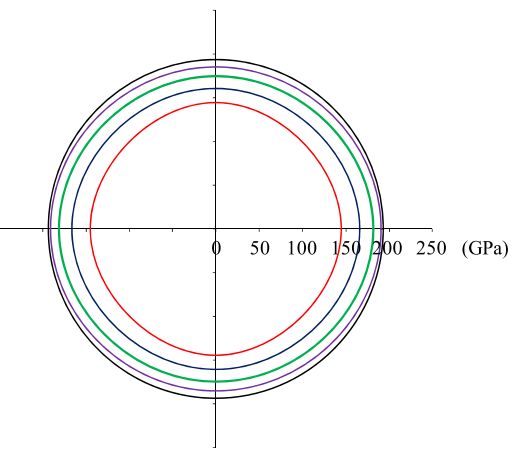

(c)

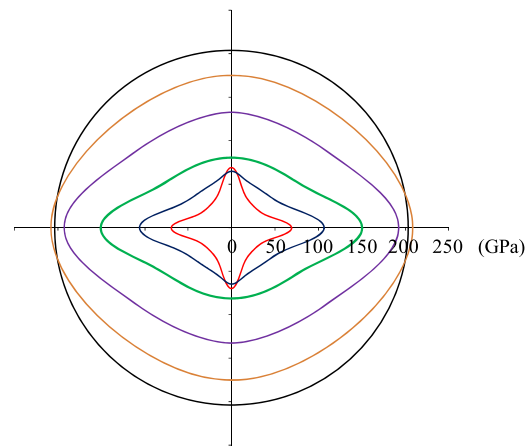

(e)

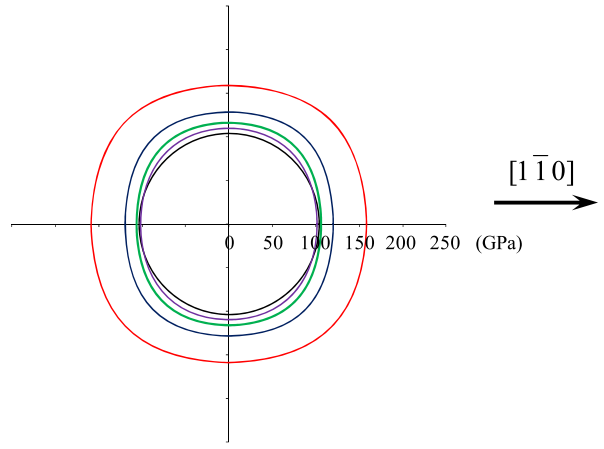

(b)

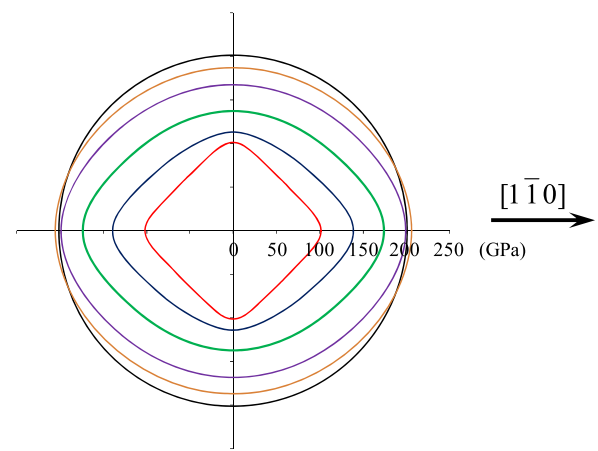

(d)

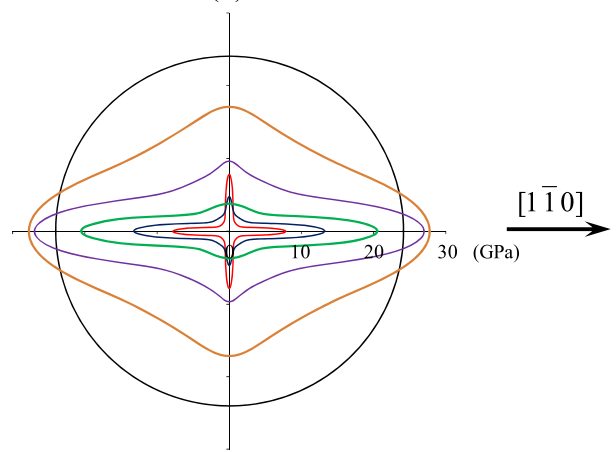

(f)

Fig. 2 Selected loci of $\tilde{E}$ for $1^{\prime}$ parallel to $[u u w]$ for (a) $\mathrm{Sm}_{0.58} \mathrm{Y}_{0.42} \mathrm{~S}$, (b) $\mathrm{Nb}$, (c) $\mathrm{Si}$, (d) $\mathrm{Cu}$, (e) $\beta$-brass and (f) In -27 at $\% \mathrm{Tl}$ at $290 \mathrm{~K}$ for various (uuw) planes between (001) and (111). In each diagram the horizontal direction is [1 10$]$. The loci are (001) in red, (113) in dark blue, (112) in green, (223) in purple, (445) in orange and (111) in black. For clarity, the (445) loci are not shown in (a), (b) and (c). (Colour figure online)

which for $\mathrm{In}-27$ at $\% \mathrm{Tl}$ at $290 \mathrm{~K}$ gives a maximum stiffness of $27.9 \mathrm{GPa}$ when $\varphi=46.9^{\circ}$, i.e., when $(u u w)$ is close to (334) and $3^{\prime}$ is parallel to [1실.

For $\theta=90^{\circ}$, these three compliance terms are

$$
s_{11}^{\prime}=s_{11}-\frac{J}{2}\left(4 \sin ^{2} \varphi-3 \sin ^{4} \varphi\right),
$$




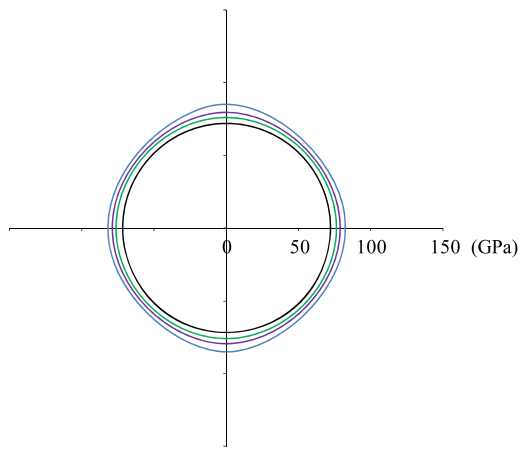

(a)

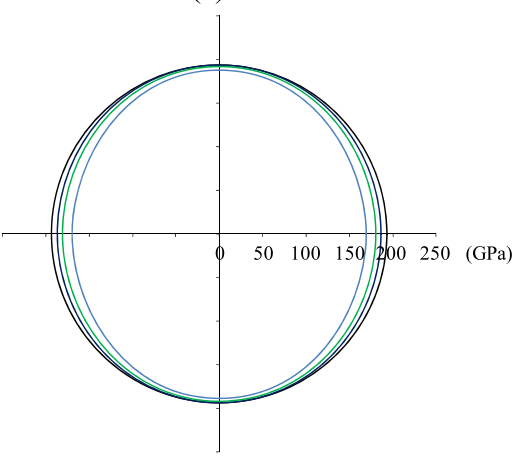

(c)

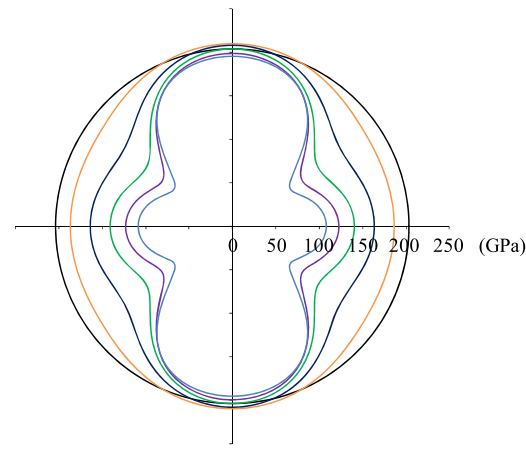

(e)

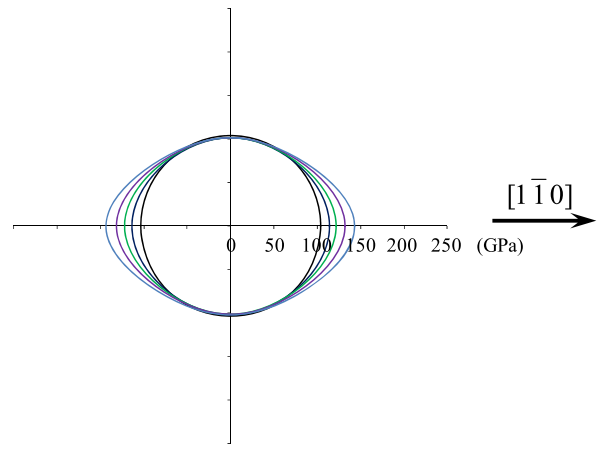

(b)

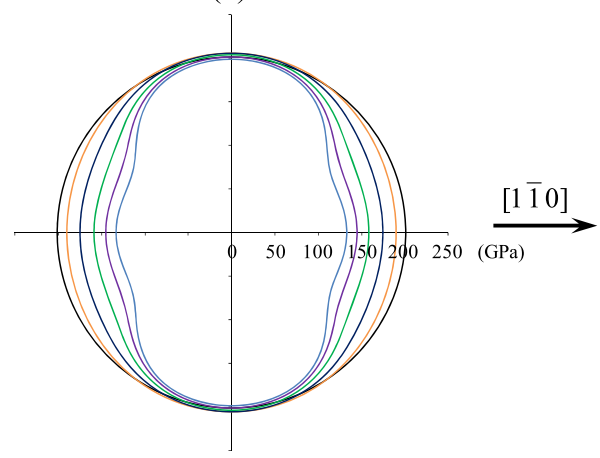

(d)

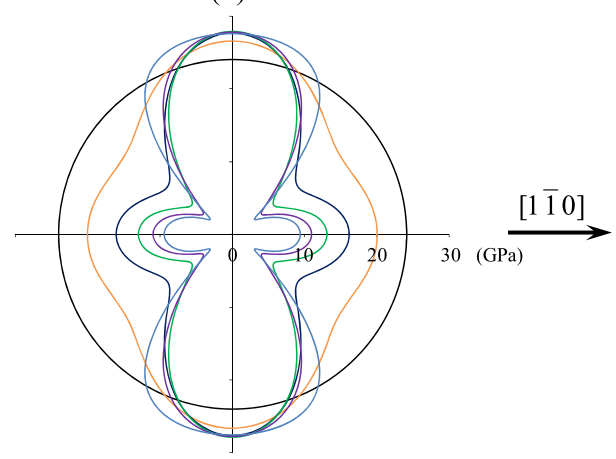

(f)

Fig. 3 Selected loci of $\tilde{E}$ for $1^{\prime}$ parallel to $[u u w]$ for (a) $\mathrm{Sm}_{0.58} \mathrm{Y}_{0.42} \mathrm{~S}$, (b) $\mathrm{Nb},(\mathbf{c}) \mathrm{Si},(\mathbf{d}) \mathrm{Cu},(\mathbf{e}) \beta$-brass and (f) $\mathrm{In}-27$ at $\% \mathrm{Tl}$ at $290 \mathrm{~K}$ for various (uuw) planes between (111) and (110). In each diagram the horizontal direction is [110]. The loci are (111) in black, (665) in orange, (332) in dark blue, (221) in green, (331) in purple and (110) in blue. For clarity, the (665) and (332) loci are not shown in (a), the (665) locus is not shown in (b) and the (665) and (331) loci are not shown in (c). (Colour figure online)

$$
\begin{aligned}
& s_{33}^{\prime}=s_{11}-\frac{J}{2}\left(1+2 \sin ^{2} \varphi-3 \sin ^{4} \varphi\right), \\
& s_{13}^{\prime}=s_{12}+\frac{3 J}{2}\left(\sin ^{2} \varphi-\sin ^{4} \varphi\right) .
\end{aligned}
$$


Hence, as when $\theta=0^{\circ}$, turning points occur in $\tilde{E}$ within the angular range $0^{\circ} \leq \varphi \leq 90^{\circ}$ when $\varphi=0^{\circ}$ and $90^{\circ}$. In addition, turning points occur when

$$
s_{13}^{\prime}+s_{33}^{\prime}=0,
$$

i.e., when

$$
\sin ^{2} \varphi=\frac{J-2 s_{11}-2 s_{12}}{J}
$$

and also when

$$
\left(s_{13}^{\prime}+2 s_{33}^{\prime}\right)-3 \sin ^{2} \varphi\left(s_{13}^{\prime}+s_{33}^{\prime}\right)=0,
$$

i.e., when

$$
\sin ^{2} \varphi=\frac{2 s_{11}+s_{12}-J}{3 s_{11}+3 s_{12}-J}=\frac{2 s_{11}+4 s_{12}+s_{44}}{4 s_{11}+8 s_{12}+s_{44}} .
$$

For materials with $A>1$, the value of $\varphi$ defined by Eq. (57) gives a minimum value of $\tilde{E}$ for those materials for which satisfy this condition. Of the four materials with $A>1$ listed in Table 1 , only $\beta$-brass and In -27 at\% Tl at $290 \mathrm{~K}$ satisfy this condition. Thus, for $\mathrm{In}-27 \mathrm{at} \% \mathrm{Tl}$ at $290 \mathrm{~K}$ when $3^{\prime}$ is perpendicular to [110] for (uuw) planes, $\tilde{E}$ has a minimum value at $\theta=90^{\circ}$ when $\varphi=33.0^{\circ}$, i.e., when (uuw) is close to (112). However, as is evident from Fig. 2(f), while this is a minimum value along the direction perpendicular to [1ํㅣㄹ, it is not an absolute minimum for $\tilde{E}$ on $(u u w)$ planes-for materials with $A>1$, this occurs on (001) planes along $\langle 100\rangle$ directions.

At the critical value of $\varphi$ defined by Eq. (59), $\tilde{E}$ simplifies after some algebra to the expression

$$
\tilde{E}=\frac{4\left(s_{11}+2 s_{12}+s_{44}\right)}{s_{44}\left(4 s_{11}+8 s_{12}+s_{44}\right)}=\frac{4 c_{44}\left(c_{11}+2 c_{12}+c_{44}\right)}{c_{11}+2 c_{12}+4 c_{44}}
$$

i.e., the same as the maximum value of $\tilde{E}$ obtained when $\theta=0^{\circ}$.

Graphical representations of how $\tilde{E}$ varies as a function of $\varphi$ within the angular range $0^{\circ} \leq \varphi \leq 90^{\circ}$ for orientations parallel and perpendicular to [1 $\left.1 \overline{1} 0\right]$ within $(u u w)$ planes for $1^{\prime}$ parallel to $[u u w]$ are shown for In -27 at\% $\mathrm{Tl}$ at $290 \mathrm{~K}$ in Fig. 4. Necessarily, these two curves intersect at the (111) orientation when $\tilde{E}$ is isotropic within the plane.

\subsection{1' Parallel to [0vw] Between [001] and [010]}

For these directions, we can parameterise $\left[a_{11}, a_{12}, a_{13}\right]$ as

$$
\left[a_{11}, a_{12}, a_{13}\right]=[0, \sin \varphi, \cos \varphi] .
$$

For each choice of $1^{\prime}$, it is now convenient to have [100] as a common horizontal direction to visualise how the loci of $\tilde{E}$ change as a function of angle $\varphi$ from [001] towards [110].

Relative to the crystallographic set of orthonormal axes $x_{1}, x_{2}$ and $x_{3}$, we can define a new orthonormal right-hand axis set $\beta_{1}, \beta_{2}$ and $\beta_{3}$, so that the table of direction cosines between the axis set 1,2 and 3 and this new axis set will be of the form 
Fig. 4 Variation of $\tilde{E}$ parallel and perpendicular to [1 $\overline{1} 0]$ within (uиw) planes for $1^{\prime}$ parallel to $[u u w]$ for $\mathrm{In}-27$ at $\% \mathrm{Tl}$ at $290 \mathrm{~K}$. The two maxima at angles of $46.9^{\circ}$ and $70.5^{\circ}$ from $(001)$ on the green and blue curves respectively are identical in magnitude. (Colour figure online)

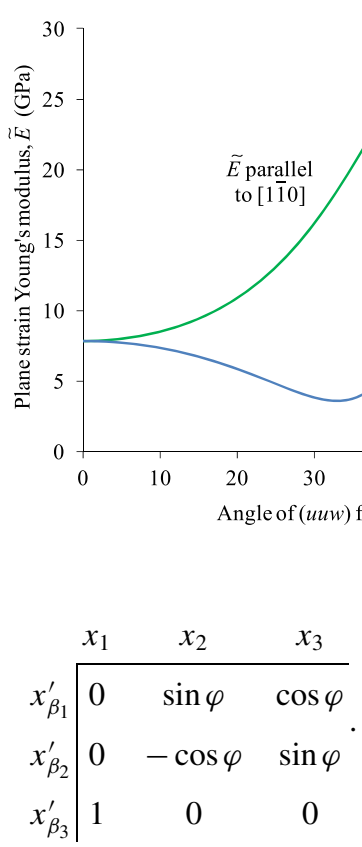

Therefore, with respect to $\beta_{2}$ and $\beta_{3}$, a direction of unit length making anticlockwise angles of $\left(90^{\circ}+\theta\right)$ with $\beta_{2}$ and $\theta$ with $\beta_{3}$ along which there is a condition of plane strain will have direction cosines

$$
\left[a_{31}, a_{32}, a_{33}\right]=[\cos \theta, \sin \theta \cos \varphi,-\sin \theta \sin \varphi] .
$$

Hence, using Eq. (9),

$$
\begin{aligned}
& s_{11}^{\prime}=s_{11}-\frac{J}{2} \sin ^{2} 2 \varphi, \\
& s_{33}^{\prime}=s_{11}-\frac{J}{2}\left(4 \sin ^{2} \theta \cos ^{2} \theta+\sin ^{4} \theta \sin ^{2} 2 \varphi\right), \\
& s_{13}^{\prime}=s_{12}+\frac{J}{2} \sin ^{2} \theta \sin ^{2} 2 \varphi .
\end{aligned}
$$

For a specific value of $\varphi$, turning points in $1 / \tilde{E}$ occur when Eq. (29) is zero, i.e., when

$$
\frac{s_{13}^{\prime} J \sin \theta \cos \theta}{s_{33}^{\prime 2}} g(\theta)=0,
$$

where

$$
g(\theta)=s_{33}^{\prime} \sin ^{2} 2 \varphi-s_{13}^{\prime}\left(\sin ^{2} \theta\left(4-\sin ^{2} 2 \varphi\right)-2\right) .
$$

The condition that $g(\theta)=0$ simplifies to the condition

$$
\sin ^{2} \theta=\frac{4 s_{12}+2 s_{11} \sin ^{2} 2 \varphi}{8 s_{12}+\left(2 s_{11}-4 s_{12}-s_{44}\right) \sin ^{2} 2 \varphi} .
$$


Therefore, within the angular range $0^{\circ} \leq \varphi \leq 90^{\circ}$ turning points occur for $\tilde{E}$ when $\theta=0^{\circ}$ and $90^{\circ}$, when $s_{13}^{\prime}=0$, and when Eq. (67) is satisfied. The symmetry of the situation shows that the loci for $45^{\circ} \leq \varphi \leq 90^{\circ}$ are mirror images of those for $0^{\circ} \leq \varphi \leq 45^{\circ}$, and so attention therefore need only be focused on $0^{\circ} \leq \varphi \leq 45^{\circ}$. Examples of loci for $(0 \mathrm{vw})$ planes for $0^{\circ}$ $\leq \varphi \leq 45^{\circ}$ are shown in Fig. 5 for the six materials for which stiffness coefficients are given in Table 1.

Thus, for In -27 at $\% \mathrm{Tl}$ at $290 \mathrm{~K}$, when $\varphi=0^{\circ}$, i.e., when [0vw] is [001], turning points occur for $\tilde{E}$ at $\theta=0^{\circ}, 45^{\circ}$ and $90^{\circ}$ within the angular range $0^{\circ} \leq \theta \leq 90^{\circ}$ because Eq. (67) is satisfied, but not the condition $s_{13}^{\prime}=0$. As $\varphi$ increases from zero, the turning point originally at $\theta=45^{\circ}$ which is a local maximum rotates very very slowly towards $\theta=0^{\circ}$, so that when $\varphi=33.7^{\circ}$ and $(0 v w)$ is $(023)$, this local maximum is at $\theta=39.8^{\circ}$ $(\tilde{E}=11.0 \mathrm{GPa})$. By this $(023)$ orientation the condition $s_{13}^{\prime}=0$ is satisfied when $\theta=64.3^{\circ}$, at which $\tilde{E}=2.7 \mathrm{GPa}$. $\tilde{E}$ is at a local maximum at $\theta=90^{\circ}(\tilde{E}=2.7 \mathrm{GPa})$ and a local minimum at $\theta=0^{\circ}(\tilde{E}=7.3 \mathrm{GPa})$ when $(0 v w)$ is $(023)$, as is evident from Fig. 5(f). In fact $\tilde{E}$ locally only becomes a maximum at $\theta=0^{\circ}$ for $\mathrm{In}-27$ at $\% \mathrm{Tl}$ at $290 \mathrm{~K}$ at values of $\varphi$ very close to (011): for $42.3^{\circ} \leq \varphi \leq 45^{\circ}$.

Both $\beta$-brass and $\mathrm{Cu}$ have $(0 v w)$ orientations at which $s_{13}^{\prime}=0$, as does $\operatorname{Sm}_{0.58} \mathrm{Y}_{0.42} \mathrm{~S}$ (discussed for the symmetrically equivalent (011) plane in Sect. 3.4), but this is not so for $\mathrm{Si}$ and $\mathrm{Nb}$ considered in Fig. 5. For all four materials with $A>1$, maxima in $\tilde{E}$ occur on the (011) plane along $\langle 100\rangle$ directions within the $(0 \mathrm{vw})$ family of planes and minima occur along the (001) plane along $\langle 110\rangle$ directions; the reverse is true for the two materials $\operatorname{Sm}_{0.58} \mathrm{Y}_{0.42} \mathrm{~S}$ and $\mathrm{Nb}$ for which $A<1$. A subtlety which occurs for $\operatorname{Sm}_{0.58} \mathrm{Y}_{0.42} \mathrm{~S}$ with $c_{12}<0$ is that as $\varphi$ increases from zero, the turning point originally at $\theta=45^{\circ}$ when $[0 v w]=[001]$, and which is a local minimum because $A<1$, rotates towards $\theta=90^{\circ}$, rather than towards $\theta=0^{\circ}$.

\subsection{Extremal Conditions for $\tilde{E}$}

The formalism introduced by Norris [9] can be used straightforwardly to obtain three conditions for a stationary value of $\tilde{E}$. Before analysing what happens to the locus of $\tilde{E}$ for the situation where $1^{\prime}$ is a general direction $[u v w]$ within the standard stereographic 001-011111 stereographic triangle, it is worthwhile considering whether or not the extreme values of $\tilde{E}$ have already been identified by the analysis in Sects. 3.1-3.6.

Applying Norris's formalism and using the equality on page 798 of his paper, it is straightforward to show that in contracted Voigt notation (see Appendix) the three conditions that an extreme value of $\tilde{E}$ must fulfil simultaneously for a general single crystalline material of triclinic symmetry are:

$$
\begin{aligned}
& s_{13}^{\prime}\left(s_{33}^{\prime} s_{14}^{\prime}-s_{13}^{\prime} s_{34}^{\prime}\right)=0, \\
& \left(s_{13}^{\prime}+s_{33}^{\prime}\right)\left(s_{33}^{\prime} s_{15}^{\prime}-s_{13}^{\prime} s_{35}^{\prime}\right)=0, \\
& s_{33}^{\prime}\left(s_{33}^{\prime} s_{16}^{\prime}-s_{13}^{\prime} s_{36}^{\prime}\right)=0 .
\end{aligned}
$$

In practice, while it can happen that $s_{13}^{\prime}=0$ and $s_{13}^{\prime}+s_{33}^{\prime}=0$ for some highly anisotropic materials, as has already been shown in Sects. 3.3-3.6, these conditions reduce to the three conditions that an extreme value of $\tilde{E}$ must fulfil simultaneously:

$$
\begin{aligned}
& \left(s_{33}^{\prime} s_{14}^{\prime}-s_{13}^{\prime} s_{34}^{\prime}\right)=0, \\
& \left(s_{33}^{\prime} s_{15}^{\prime}-s_{13}^{\prime} s_{35}^{\prime}\right)=0,
\end{aligned}
$$




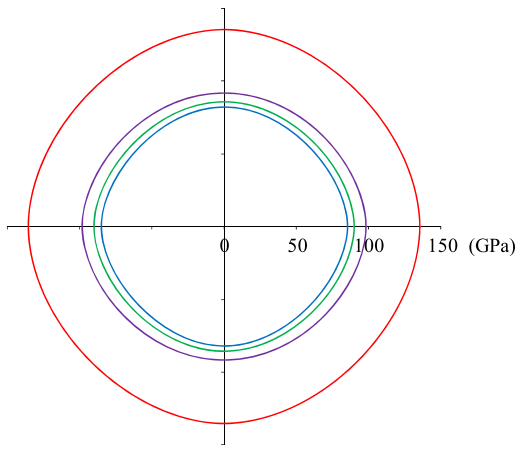

(a)

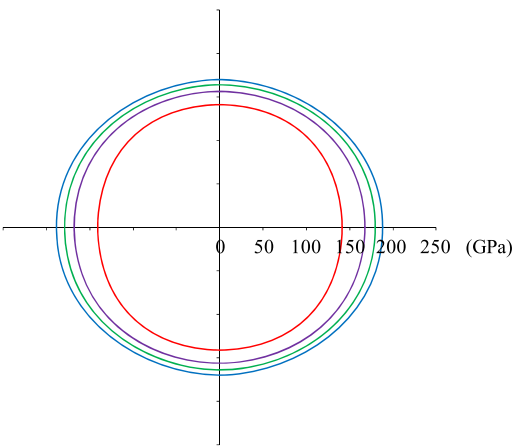

(c)

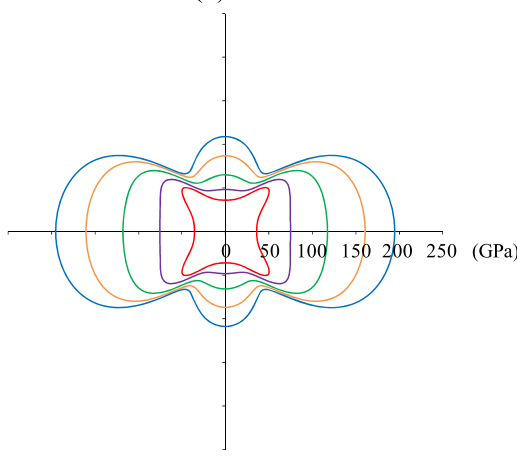

(e)

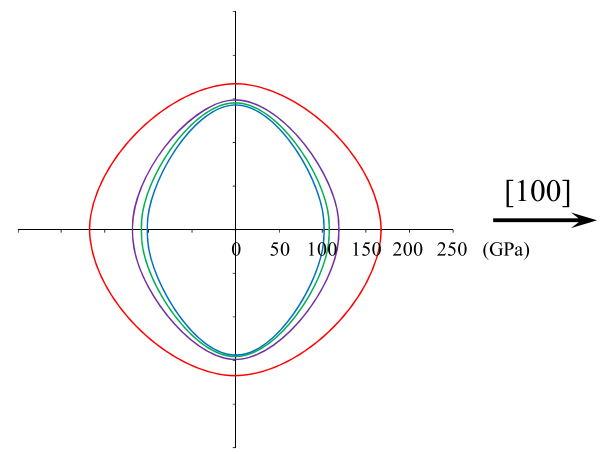

(b)

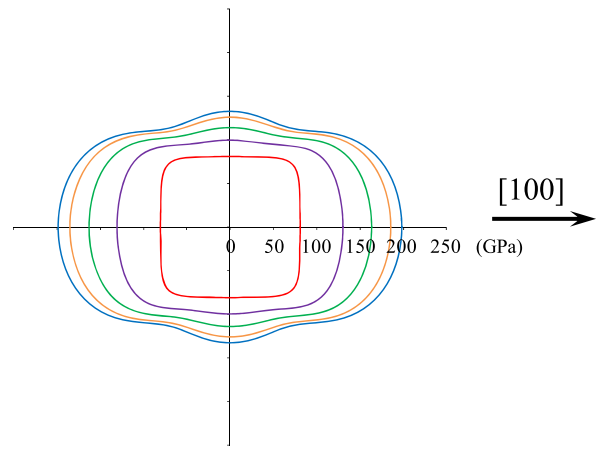

(d)

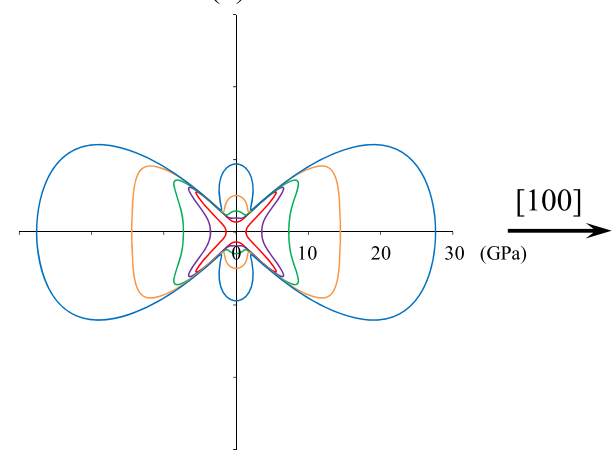

(f)

Fig. 5 Selected loci of $\tilde{E}$ for $1^{\prime}$ parallel to $[0 v w]$ for (a) $\mathrm{Sm}_{0.58} \mathrm{Y}_{0.42} \mathrm{~S}$, (b) $\mathrm{Nb}$, (c) $\mathrm{Si}$, (d) $\mathrm{Cu},(\mathbf{e}) \beta$-brass and (f) $\mathrm{In}-27$ at $\% \mathrm{Tl}$ at $290 \mathrm{~K}$ for various $(0 \mathrm{vw})$ planes between $(001)$ and (011). In each diagram the horizontal direction is [100]. The loci are (001) in red, (012) in purple, (023) in green, (045) in orange and (011) in blue. For clarity, the (045) loci are not shown in (a), (b) and (c). (Colour figure online)

$$
\left(s_{33}^{\prime} s_{16}^{\prime}-s_{13}^{\prime} s_{36}^{\prime}\right)=0 .
$$

For cubic materials, it is evident using Eq. (8) that the new $s_{i j}^{\prime}$ that now need to be considered are

$$
s_{14}^{\prime}=2 S_{1123}^{\prime}=2 J\left(a_{11}^{2} a_{21} a_{31}+a_{12}^{2} a_{22} a_{32}+a_{13}^{2} a_{23} a_{33}\right),
$$




$$
\begin{aligned}
& s_{34}^{\prime}=2 S_{3323}^{\prime}=2 J\left(a_{21} a_{31}^{3}+a_{22} a_{32}^{3}+a_{23} a_{33}^{3}\right), \\
& s_{15}^{\prime}=2 S_{1131}^{\prime}=2 J\left(a_{11}^{3} a_{31}+a_{12}^{3} a_{32}+a_{13}^{3} a_{33}\right), \\
& s_{35}^{\prime}=2 S_{3331}^{\prime}=2 J\left(a_{11} a_{31}^{3}+a_{12} a_{32}^{3}+a_{13} a_{33}^{3}\right), \\
& s_{16}^{\prime}=2 S_{1112}^{\prime}=2 J\left(a_{11}^{3} a_{21}+a_{12}^{3} a_{22}+a_{13}^{3} a_{23}\right), \\
& s_{36}^{\prime}=2 S_{3312}^{\prime}=2 J\left(a_{11} a_{21} a_{31}^{2}+a_{12} a_{22} a_{32}^{2}+a_{13} a_{23} a_{33}^{2}\right),
\end{aligned}
$$

all of which are identically equal to zero if the cubic material is isotropic, i.e., if $A=1$ and $J=0$. Under such conditions, Eqs. (71)-(73) are clearly satisfied simultaneously.

For $A \neq 1$, suppose first that $[u v w]$ is $[0 v w]$ so that the table of direction cosines between the axis set 1,2 and 3 and the new axis set $1^{\prime}, 2^{\prime}, 3^{\prime}$ can be taken to be of the form

\begin{tabular}{c|ccc}
\multicolumn{1}{c}{$x_{1}$} & $x_{2}$ & $x_{3}$ \\
\cline { 2 - 4 }$x_{1}^{\prime}$ & 0 & $\sin \varphi$ & $\cos \varphi$ \\
$x_{2}^{\prime}$ & $\sin \theta$ & $-\cos \theta \cos \varphi$ & $\cos \theta \sin \varphi$ \\
$x_{3}^{\prime}$ & $\cos \theta$ & $\sin \theta \cos \varphi$ & $-\sin \theta \sin \varphi$
\end{tabular}.

Under these circumstances,

$$
\begin{aligned}
& s_{14}^{\prime}=-\frac{J}{2} \sin 2 \theta \sin ^{2} 2 \varphi, \\
& s_{34}^{\prime}=\frac{J}{2} \sin 2 \theta\left(2-\sin ^{2} \theta\left(4-\sin ^{2} 2 \varphi\right)\right), \\
& s_{15}^{\prime}=-\frac{J}{2} \sin \theta \sin 4 \varphi, \\
& s_{35}^{\prime}=\frac{J}{2} \sin ^{3} \theta \sin 4 \varphi \\
& s_{16}^{\prime}=\frac{J}{2} \cos \theta \sin 4 \varphi \\
& s_{36}^{\prime}=-\frac{J}{2} \cos \theta \sin ^{2} \theta \sin 4 \varphi
\end{aligned}
$$

Clearly, Eqs. (71)-(73) are satisfied simultaneously if each of these terms is zero. Hence, if $\varphi=0^{\circ}$ or $90^{\circ}, s_{14}^{\prime}=s_{15}^{\prime}=s_{35}^{\prime}=s_{16}^{\prime}=s_{36}^{\prime}=0$ irrespective of the value of $\theta$, while $s_{34}^{\prime}=0$ at these values of $\varphi$ only when $\theta=45^{\circ} n$ for $n$ integer. Hence it can be concluded that if [uvw] is [001], values of $\theta=45^{\circ} n$ for $n$ integer are potential candidates to consider for global extrema of $\tilde{E}$, i.e., the $\langle 100\rangle$ and $\langle 110\rangle$ directions within (001).

If $\varphi=45^{\circ}, s_{15}^{\prime}=s_{35}^{\prime}=s_{16}^{\prime}=s_{36}^{\prime}=0$ irrespective of the value of $\theta$, while $s_{14}^{\prime}=s_{34}^{\prime}=0$ when $\theta=90^{\circ} n$ for $n$ integer. Therefore, it can be concluded that if [uvw] is [011], values of $\theta=90^{\circ} n$ for $n$ integer are also potential candidates to consider for global extrema of $\tilde{E}$, i.e., the $\langle 100\rangle$ and $\langle 0 \overline{1} 1\rangle$ directions within $(011)$.

For all other $[0 \mathrm{vw}]$ orientations, it is not possible to make each of these six terms zero simultaneously, nor is it possible to satisfy Eqs. (71)-(73) otherwise, but it is of interest to note that the condition

$$
\left(s_{33}^{\prime} s_{14}^{\prime}-s_{13}^{\prime} s_{34}^{\prime}\right)=0,
$$


is equivalent to the condition for a turning point expressed by $g(\theta)=0$ where $g(\theta)$ is given by Eq. (66). However, for a particular [0vw] for which Eq. (71) is satisfied for a particular combination of $\theta$ and $\varphi$, Eq. (72) and Eq. (73) will not be satisfied, i.e., it does not define a potential candidate for global extrema of $\tilde{E}$.

The insight gained into the consideration of possible global extrema of $\tilde{E}$ when $1^{\prime}$ is of the form $[0 v w]$ is very helpful in establishing possible global extrema of $\tilde{E}$ when $1^{\prime}$ is of the form $[u u w]$. If we consider the table of direction cosines

$$
\begin{array}{c|ccc}
\multicolumn{1}{c}{} & x_{1} & x_{2} & x_{3} \\
\cline { 2 - 4 } x_{1}^{\prime} & \frac{\sin \varphi}{\sqrt{2}} & \frac{\sin \varphi}{\sqrt{2}} & \cos \varphi \\
x_{2}^{\prime} & \frac{-\cos \varphi}{\sqrt{2}} & \frac{-\cos \varphi}{\sqrt{2}} & \sin \varphi \\
x_{3}^{\prime} & \frac{1}{\sqrt{2}} & -\frac{1}{\sqrt{2}} & 0
\end{array}
$$

it is evident that $s_{14}^{\prime}=s_{34}^{\prime}=s_{15}^{\prime}=s_{35}^{\prime}=0$, and so Eqs. (71) and (72) are satisfied. $s_{33}^{\prime}, s_{16}^{\prime}$, $s_{13}^{\prime}$ and $s_{36}^{\prime}$ are given by the expressions

$$
\begin{aligned}
& s_{33}^{\prime}=s_{11}-\frac{J}{2}, \\
& s_{16}^{\prime}=\frac{J}{2} \sin 2 \varphi\left(2-3 \sin ^{2} \varphi\right), \\
& s_{13}^{\prime}=s_{12}+\frac{J}{2} \sin ^{2} \varphi, \\
& s_{36}^{\prime}=-\frac{J}{2} \sin 2 \varphi,
\end{aligned}
$$

so that Eq. (73) becomes the condition

$$
J \sin 2 \varphi\left\{\left(s_{11}-\frac{J}{2}\right)\left(2-3 \sin ^{2} \varphi\right)+\left(s_{12}+\frac{J}{2} \sin ^{2} \varphi\right)\right\}=0 .
$$

The $\sin 2 \varphi$ term outside the curly bracket indicates that, as expected from the analysis for when $1^{\prime}$ is of the form $[0 v w], \varphi=45^{\circ}$ enables Eq. (73) to be satisfied, i.e., when $[0 v w]$ is [011]. In addition, the term within the curly brackets rearranges to a condition for $\sin ^{2} \varphi$ :

$$
\sin ^{2} \varphi=\frac{2 s_{11}+s_{12}-J}{3 s_{11}-2 J} \equiv \frac{2 s_{11}+4 s_{12}+s_{44}}{2\left(s_{11}+2 s_{12}+s_{44}\right)},
$$

which is Eq. (53). Hence, Eqs. (71)-(73) are also satisfied globally when $3^{\prime}$ is the [1 10$]$ direction at the angle $\varphi$ for which $\tilde{E}$ is known to be a maximum on (uuw) planes when $A>1$ or a minimum if $A<1$.

If now for $1^{\prime}$ parallel to $[u u w]$ we consider the table of direction cosines

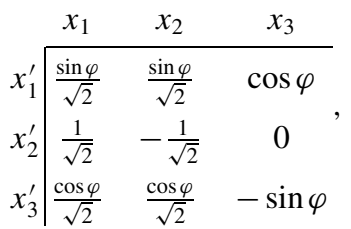


so that within (uuw) $3^{\prime}$ is perpendicular to [1ํㅣㄹ, it is evident that $s_{14}^{\prime}=s_{34}^{\prime}=s_{16}^{\prime}=s_{36}^{\prime}=0$, and so Eqs. (71) and (73) are satisfied. $s_{15}^{\prime}$ and $s_{35}^{\prime}$ are now given by the expressions

$$
\begin{aligned}
& s_{15}^{\prime}=\frac{J}{2} \sin 2 \varphi\left(3 \sin ^{2} \varphi-2\right), \\
& s_{35}^{\prime}=\frac{J}{2} \sin 2 \varphi\left(1-3 \sin ^{2} \varphi\right) .
\end{aligned}
$$

Hence, Eq. (72) becomes the condition

$$
J \sin 2 \varphi\left\{s_{33}^{\prime}\left(3 \sin ^{2} \varphi-2\right)-s_{13}^{\prime}\left(1-3 \sin ^{2} \varphi\right)\right\}=0,
$$

where the forms of $s_{33}^{\prime}$ and $s_{13}^{\prime}$ are given in Eq. (55). As expected, the condition $\sin 2 \varphi=0$ enables Eq. (72) to be satisfied when $[u u w]$ is either [001] or [110]. In addition, the term within the curly brackets rearranges to the condition:

$$
\left(s_{13}^{\prime}+2 s_{33}^{\prime}\right)-3 \sin ^{2} \varphi\left(s_{13}^{\prime}+s_{33}^{\prime}\right)=0,
$$

which is Eq. (58). Therefore, Eqs. (71)-(73) are also satisfied globally when $3^{\prime}$ is perpendicular to the [1 10$]$ direction at the angle $\varphi$ defined by Eq. (59) for which $\tilde{E}$ is known to be a maximum on (uuw) planes when $A>1$, or minimum if $A<1$.

\subsection{1' Parallel to $[u v w]$ Within the Standard Stereographic Triangle}

It is evident from the considerations in Sect. 3.7 that it is actually very difficult to satisfy Eqs. (71)-(73) simultaneously for the relatively special cases of $1^{\prime}$ of the forms $[0 v w]$ and $[u u w]$. For more general $[u v w]$ forms these conditions are sufficiently restrictive that while conditions can be identified for which there are extrema for $\tilde{E}$ within a given $(u v w)$ plane, there will not be any combinations of $1^{\prime}$ and $3^{\prime}$ for which Eqs. (71)-(73) are satisfied simultaneously.

As examples of the general principles for more general $[u v w]$, numerical calculations have been undertaken for In -27 at $\% \mathrm{Tl}$ at $290 \mathrm{~K}$, as this shows most obviously the criteria which determine the local maxima and minima of $\tilde{E}$ for a particular $(u v w)$. Loci of how $\tilde{E}$ varies within the (156), (345) and (125) planes are shown in Fig. 6(a)-(c) respectively. For each of these graphical representations, the vertical axis has been chosen so that it is the irrational direction within $(u v w)$ along which $\tilde{E}$ is an absolute maximum. Each graphical representation only has the symmetry of a diad at the origin; the perpendicular mirror planes evident on the graphical representations of $\tilde{E}$ for $(0 v w)$ and $(u u w)$ are absent on these more general $(u v w)$ representations.

The numerical calculations show that along this irrational direction the condition

$$
\left(s_{33}^{\prime} s_{14}^{\prime}-s_{13}^{\prime} s_{34}^{\prime}\right)=0
$$

is satisfied, i.e., Eq. (71). In Fig. 6(a) this direction is $0.46^{\circ}$ clockwise relative to [4리] Fig. 6(b) this direction is $0.38^{\circ}$ anticlockwise relative to [4 $\left.\overline{3} 0\right]$, and in Fig. 6(c) this direction is $2.52^{\circ}$ anticlockwise relative to [5피].

In Fig. 6(a) a second orientation for which this condition is also satisfied lies at a conventional anticlockwise rotation of orientation of $21.87^{\circ}$ relative to the horizontal axis; this 


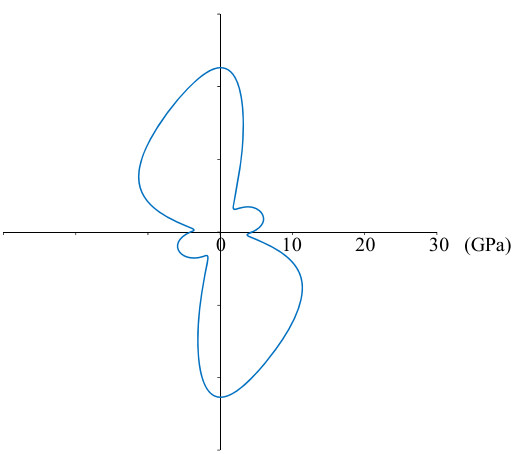

(a)

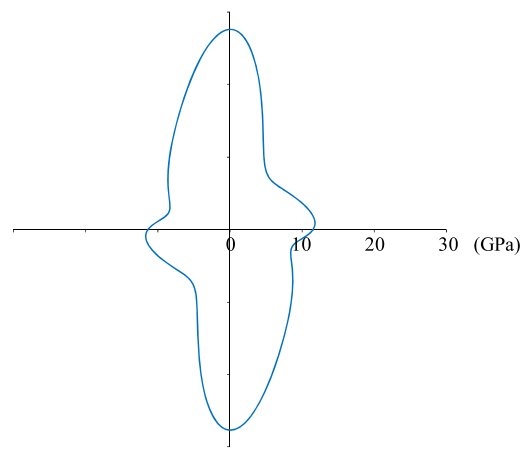

(b)

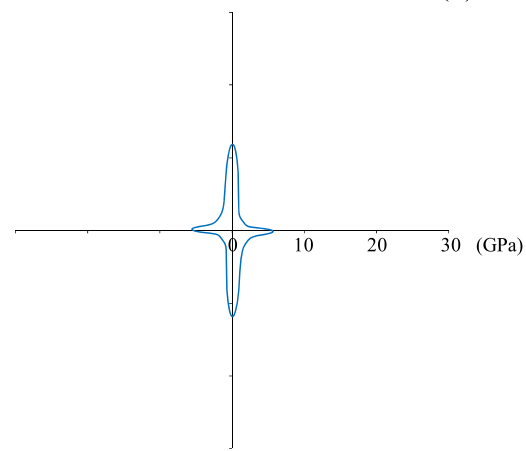

(c)

Fig. 6 Loci of $\tilde{E}$ for $1^{\prime}$ parallel to (a) [156], (b) [345] and (c) [125] for In -27 at\% Tl at $290 \mathrm{~K}$. In each graphical representation the maximum value of $\tilde{E}$ is shown vertically. In (a) the vertical direction is close to

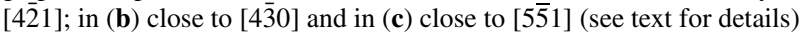

is a local maximum in $\tilde{E}$. Minima in the magnitude of $\tilde{E}$ on (156) occur at angles of $59.89^{\circ}$ and $174.94^{\circ}$ relative to the horizontal axis. At these minima, $s_{13}^{\prime}=0$. Of particular note is the fact that the angle between the positions satisfying Eq. (71) in the (156) plane are not perpendicular to one another, unlike for the $(0 v w)$ and $(u u w)$ planes.

Similar principles apply when interpreting the locus of $\tilde{E}$ on (345): in Fig. (b) the positions satisfying Eq. (71) are at orientations of $5.22^{\circ}$ and $90^{\circ}$ relative to the horizontal axis, while the minima at orientations of $47.98^{\circ}$ and $162.88^{\circ}$ satisfy the condition $s_{13}^{\prime}=0$. In Fig. 6(c) there are four positions satisfying Eq. (71) at orientations of $36.86^{\circ}, 90^{\circ}, 142.40^{\circ}$ and $178.42^{\circ}$ relative to the horizontal axis; there are no positions for which $s_{13}^{\prime}=0$ is satisfied.

Summarising, within a general plane of the form (uvw), extrema for $\tilde{E}$ occur where either $\left(s_{33}^{\prime} s_{14}^{\prime}-s_{13}^{\prime} s_{34}^{\prime}\right)=0$ or $s_{13}^{\prime}=0$. This statement applies irrespective of whether or not the pole of $(u v w)$ is in the standard stereographic triangle. For most materials the condition $s_{13}^{\prime}=0$ is not satisfied - materials in the form of single crystals which satisfy this fairly restrictive condition have to be quite anisotropic in terms of their elastic properties. Finally, none of the extrema for $\tilde{E}$ on general ( $u v w)$ planes are candidates for global extrema-it is now apparent from examining the loci of $\tilde{E}$ on general (uvw) planes that these global extrema in $\tilde{E}$ arise on planes of the form $\{001\}$ and $\{u u w\}$, as discussed in Sect. 3.7. 


\section{Discussion and Conclusions}

Although the concept of plane strain is useful in a number of physically significant situations within elasticity, in particular elastic contact problems and the bending of short, relatively wide beams, it is perhaps somewhat surprising that the consequences of elastic anisotropy when dealing with cubic single crystals subjected to plane strain are not already well established in the literature.

Analysis of how the plane strain Young's modulus $\tilde{E}$ varies as a function of direction $1^{\prime}$ along which the uniaxial stress is applied and the perpendicular direction $3^{\prime}$ along which conditions of plane strain are maintained during the elastic deformation process shows that there are a relatively small number of simple guiding principles. For materials with $A>1$, minima in $\tilde{E}$ occur when $1^{\prime}$ is along a $\langle 001\rangle$ direction and $3^{\prime}$ along a perpendicular $\langle 100\rangle$ direction; maxima in $\tilde{E}$ occur when $1^{\prime}$ is along a $\langle u u w\rangle$ direction and $3^{\prime}$ is either along a perpendicular $\langle 1 \overline{1} 0\rangle$ direction or a $\langle w w \overline{2 u}\rangle$ direction. Results for materials with $A<1$ are the opposite of those for $A>1$.

Thus, to take the specific example of silicon, the minimum value of $\tilde{E}$ is $144.7 \mathrm{GPa}$ when the applied stress is along $\langle 001\rangle$ and plane strain conditions are maintained on $\{100\}$ planes containing the direction of applied stress. From Eqs. (53), (54), (59) and (60), the maximum value of $\tilde{E}$ of $194.1 \mathrm{GPa}$ occurs when (i) $1^{\prime}$ is along [uuw] 51.2 away from [001] and $3^{\prime}$ is along [1 10$]$ and (ii) $1^{\prime}$ is along $[u u w] 59.3^{\circ}$ away from [001] and $3^{\prime}$ is along $[w w \overline{2 u}]$. For silicon this maximum value of $\tilde{E}$ compares with a value of $\tilde{E}$ of $193.5 \mathrm{GPa}$ when $1^{\prime}$ is along [111], 54.74 from [001] (Eq. (17)), where $3^{\prime}$ need only be specified as being perpendicular to $1^{\prime}$ because under these circumstances the locus of $\tilde{E}$ is a circle. Not surprisingly from a consideration of these data, the orientations of $1^{\prime}$ at which $\tilde{E}$ is at a maximum for silicon are very close to [111]. The practical consequence of these calculations for silicon, a material widely available in single crystalline form and used for microelectromechanical systems, are that wide beams with $1^{\prime}$ along a $\langle 111\rangle$ direction can be up to $33 \%$ stiffer in their plane strain Young's modulus than beams where $1^{\prime}$ is along $\langle 001\rangle$.

The other range of physical situations in which plane strain conditions are encountered when dealing with isotropic materials are contact problems. The contact problem for isotropic bodies originally solved by Hertz [26] is one well-known contact problem. This problem is relevant to the nanoindentation of isotropic materials when axisymmetric indenters are used. The sum of the displacements of the indenter and material being indented is proportional to the sum of the reciprocals of the plane strain Young's moduli of the two materials [1]. Knowing this, in nanoindentation, the contact stiffness, $S_{\mathrm{c}}$, measured on unloading can be shown to be proportional to the product of the square root of the projected contact area, $A_{p}$, and what is termed the 'reduced modulus', $E_{r}$ :

$$
\frac{1}{E_{r}}=\frac{1-v_{s}^{2}}{E_{I T}}+\frac{1-v_{i}^{2}}{E_{i}},
$$

where $E_{I T}$ is the indentation modulus of the specimen and the subscripts $s$ and $i$ denotes 'specimen' and 'indenter' respectively, so that overall

$$
S_{\mathrm{c}}=\frac{2}{\sqrt{\pi}} E_{r} \sqrt{A_{p}}
$$

[27, 28]. Work by Vlassak and Nix [29] has shown that a formula equivalent to Eq. (84) arises when considering the indentation of the more complex problem of anisotropic materials, but that the indentation modulus determined in this manner will depend on the actual 
indenter geometry, unless conditions of high symmetry pertain, e.g., when the contact area between the indenter and the material being indented is circular and when the indented material has a three-fold or four-fold axis parallel to the direction of loading. More recent work by Vlassak and co-workers [30] has enabled 'equivalent indentation moduli' to be determined for elastically anisotropic materials, but it is evident that these contact problems cease to be problems of plane strain when dealing with elastically anisotropic materials.

Instead, results from this analysis are relevant to plane-strain compression tests of cubic single crystals. Plane-strain compression tests are usually carried out in the context of analysing metalworking processes such as cold rolling, in which plastic deformation is deliberately introduced [31]. However, such tests in the elastic regime on suitably prepared cubic single crystals would be expected to exhibit the variations in plane strain Young's modulus as a function of loading direction and direction under which plane strain conditions pertain that have been determined in this paper.

Open Access This article is distributed under the terms of the Creative Commons Attribution 4.0 International License (http://creativecommons.org/licenses/by/4.0/), which permits unrestricted use, distribution, and reproduction in any medium, provided you give appropriate credit to the original author(s) and the source, provide a link to the Creative Commons license, and indicate if changes were made.

\section{Appendix: The Voigt Notation}

In elasticity, the symmetric stress and strain tensors of the second rank, $\sigma_{i j}$ and $\varepsilon_{k l}$ respectively, are related to one another through the equations

$$
\sigma_{i j}=C_{i j k l} \varepsilon_{k l} \quad \text { and } \quad \varepsilon_{i j}=S_{i j k l} \sigma_{k l} \text {, }
$$

in which $C_{i j k l}$ is the stiffness tensor and $S_{i j k l}$ is the compliance tensor, both tensors of the fourth rank, and where $i, j, k$ and $l$ take all values between 1 and 3 [5, 7, 8, 12, 13].

The Voigt notation is a convenient contracted notation to express these tensor relations in matrix form. In this notation, the symmetrical stress tensor $\sigma_{i j}$ is contracted as follows:

$$
\left(\begin{array}{lll}
\sigma_{11} & \sigma_{12} & \sigma_{13} \\
\sigma_{12} & \sigma_{22} & \sigma_{23} \\
\sigma_{13} & \sigma_{23} & \sigma_{33}
\end{array}\right) \rightarrow\left(\begin{array}{lll}
\sigma_{1} & \sigma_{6} & \sigma_{5} \\
\sigma_{6} & \sigma_{2} & \sigma_{4} \\
\sigma_{5} & \sigma_{4} & \sigma_{3}
\end{array}\right)
$$

A similar procedure applies to the symmetrical strain tensor $\varepsilon_{i j}$, but here it is necessary to introduce factors of $\frac{1}{2}$ :

$$
\left(\begin{array}{lll}
\varepsilon_{11} & \varepsilon_{12} & \varepsilon_{13} \\
\varepsilon_{12} & \varepsilon_{22} & \varepsilon_{23} \\
\varepsilon_{13} & \varepsilon_{23} & \varepsilon_{33}
\end{array}\right) \rightarrow\left(\begin{array}{ccc}
\varepsilon_{1} & \frac{1}{2} \varepsilon_{6} & \frac{1}{2} \varepsilon_{5} \\
\frac{1}{2} \varepsilon_{6} & \varepsilon_{2} & \frac{1}{2} \varepsilon_{4} \\
\frac{1}{2} \varepsilon_{5} & \frac{1}{2} \varepsilon_{4} & \varepsilon_{3}
\end{array}\right) .
$$

These contracted forms for the stress and strain tensors are then related to one another through the two equations

$$
\sigma_{m}=c_{m n} \varepsilon_{n}
$$

and

$$
\varepsilon_{m}=s_{m n} \sigma_{n}
$$


where $m$ and $n$ take all values from 1 to 6 and where $c_{m n}$ and $s_{m n}$ are the contracted forms of the $C_{i j k l}$ and $S_{i j k l}$ respectively. In these contracted forms, the $i$ and $j$ values of $1,2,3,4,5$, 6 correspond to the six pairs 11, 22, 33, 23, 31, 12 in the full tensor notation. This notation is able to be used because the stiffness and compliance tensors both satisfy the condition

$$
T_{i j k l}=T_{i j l k}=T_{j i k l}=T_{j i l k}
$$

for a tensor $T_{i j k l}$ of the fourth rank.

While writing the $C_{i j k l}$ in terms of their corresponding $c_{m n}$ is straightforward, so that, for example, $C_{1233} \rightarrow c_{63}, C_{2323} \rightarrow c_{44}, C_{2332} \rightarrow c_{44}$, etc., factors of 2 and 4 have to be introduced into the definition of $s_{m n}$, so that

$$
s_{m n}=\frac{4 S_{i j k l}}{\left(1+\delta_{i j}\right)\left(1+\delta_{k l}\right)},
$$

where $\delta_{i j}$ is the Kronecker delta. Thus, for example, $s_{13}=S_{1133}, s_{14}=2 S_{1123}$ and $s_{46}=$ $4 S_{2312}$. Since the energy stored in an elastically strained crystal depends on the strain attained, and not on the path by which the strained state is reached, it follows that $c_{m n}=c_{n m}$ and $s_{m n}=s_{n m}$.

In cubic crystals when the axis system is parallel to the crystal axes, there are only three independent elastic constants: $c_{11}=c_{22}=c_{33}, c_{12}=c_{23}=c_{31}$ and $c_{44}=c_{55}=c_{66}$, written conventionally as $c_{11}, c_{12}$ and $c_{44}$. An equivalent result holds for the compliance constants $s_{11}, s_{12}$ and $s_{44}$; these are related to $c_{11}, c_{12}$ and $c_{44}$ through the equations:

$$
\begin{aligned}
& s_{11}=\frac{c_{11}+c_{12}}{\left(c_{11}-c_{12}\right)\left(c_{11}+2 c_{12}\right)}, \\
& s_{12}=-\frac{c_{12}}{\left(c_{11}-c_{12}\right)\left(c_{11}+2 c_{12}\right)}, \\
& s_{44}=\frac{1}{c_{44}} .
\end{aligned}
$$

\section{References}

1. Timoshenko, S.P., Goodier, J.N.: Theory of Elasticity 3rd edn. pp. 15-17. McGraw-Hill, New York (1987)

2. Johnson, K.L.: Contact Mechanics. Cambridge University Press, Cambridge (1985)

3. Young, W.C.: Roark's Formulas for Stress and Strain, 6th edn. p. 204. McGraw-Hill, New York (1989)

4. Hopcroft, M.A., Nix, W.D., Kenny, T.W.: What is the Young's modulus of silicon? J. Microelectromech. Syst. 19, 229-238 (2010)

5. Bower, A.F.: Applied Mechanics of Solids. CRC Press, Boca Raton (2009)

6. Lawn, B.R.: Fracture of Brittle Solids, 2nd edn. p. 8. Cambridge University Press, Cambridge (1993)

7. Ting, T.C.T.: Anisotropic Elasticity: Theory and Applications. Oxford University Press, Oxford (1996)

8. Nye, J.F.: Physical Properties of Crystals, pp. 131-149. Clarendon Press, Oxford (1985)

9. Norris, A.N.: Extreme values of Poisson's ratio and other engineering moduli in anisotropic materials. J. Mech. Mater. Struct. 1, 793-812 (2006)

10. Norris, A.N.: Poisson's ratio in cubic materials. Proc. R. Soc. A 462, 3385-3405 (2006)

11. Brańka, A.C., Heyes, D.M., Wojciechowski, K.W.: Auxeticity of cubic materials. Phys. Status Solidi B, Basic Solid State Phys. 246, 2063-2071 (2009)

12. Voigt, W.: Lehrbuch der Kristallphysik. Teubner, Leipzig (1910)

13. Knowles, K.M.: The biaxial moduli of cubic materials subjected to an equi-biaxial elastic strain. J. Elast. 124, 1-25 (2016) 
14. Hirth, J.P., Lothe, J.: Theory of Dislocations, 2nd edn. Wiley, New York (1982)

15. Zener, C.: Elasticity and Anelasticity of Metals. University of Chicago Press, Chicago (1948)

16. Kelly, A., Knowles, K.M.: Crystallography and Crystal Defects, 2nd edn. Wiley, Chichester (2012)

17. Gunton, D.J., Saunders, G.A.: Stability limits on the Poisson ratio: application to a martensitic transformation. Proc. R. Soc. Lond. A 343, 63-83 (1975)

18. Grimvall, G.: Thermophysical Properties of Materials. Elsevier, Amsterdam (1999)

19. Boppart, H., Treindl, A., Wachter, P., Roth, S.: First observation of a negative elastic constant in intermediate valent TmSe. Solid State Commun. 35, 483-486 (1980)

20. Boppart, H., Rehwald, W., Kaldis, E., Wachter, P.: Study about the onset of intermediate valency in $\mathrm{TmSe}_{0.32} \mathrm{Te}_{0.68}$ under pressure. Physica B 117, 573-575 (1983)

21. Hailing, T., Saunders, G.A., Yoğurţu, Y.K., Bach, H., Methfessel, S.: Poisson's ratio limits and effects of hydrostatic pressure on the elastic behaviour of $\mathrm{Sm}_{1-x} \mathrm{Y}_{x} \mathrm{~S}$ alloys in the intermediate valence state. J. Phys. C, Solid State Phys. 17, 4559-4573 (1984)

22. Schärer, U., Wachter, P.: Negative elastic constants in intermediate valent $\mathrm{Sm}_{x} \mathrm{La}_{1-x} \mathrm{~S}$. Solid State Commun. 96, 497-501 (1995)

23. Schärer, U., Jung, A., Wachter, P.: Brillouin spectroscopy with surface acoustic waves on intermediate valent, doped SmS. Physica B 244, 148-153 (1998)

24. Mook, H.A., Nicklow, R.M., Penney, T., Holtzberg, F., Shafer, M.W.: Phonon dispersion in intermediatevalence $\mathrm{Sm}_{0.75} \mathrm{Y}_{0.25}$ S. Phys. Rev. B 18, 2925-2928 (1978)

25. Lazarus, D.: The variation of the adiabatic elastic constants of $\mathrm{KCl}, \mathrm{NaCl}, \mathrm{CuZn}, \mathrm{Cu}$, and $\mathrm{Al}$ with pressure to 10,000 bars. Phys. Rev. 76, 545-553 (1949)

26. Hertz, H.: Ueber die Berührung fester elastischer Körper. J. Reine Angew. Math. 92, 156-171 (1881)

27. Oliver, W.C., Pharr, G.M.: An improved technique for determining hardness and elastic modulus using load and displacement sensing indentation experiments. J. Mater. Res. 7, 1564-1583 (1992)

28. Fischer-Cripps, A.C.: Nanoindentation, 3rd edn. Springer, New York/Heidelberg (2011)

29. Vlassak, J.J., Nix, W.D.: Indentation modulus of elastically anisotropic half spaces. Philos. Mag. A 67, $1045-1056$ (1993)

30. Vlassak, J.J., Ciavarella, M., Barber, J.R., Wang, X.: The indentation modulus of elastically anisotropic materials for indenters of arbitrary shape. J. Mech. Phys. Solids 51, 1701-1721 (2003)

31. Watts, A.B., Ford, H.: On the basic yield curve for a metal. Proc. Inst. Mech. Eng. 169, 1141-1149 (1955); Communications and Authors' Reply: 1150-1156 